\title{
Homeostatic Depression Shows Heightened Sensitivity to Synaptic Calcium
}

\section{OPEN ACCESS}

Edited by:

Mathieu Letellier, UMR5297 Institut Interdisciplinaire de Neurosciences (IINS), France

Reviewed by: Dion Dickman, University of Southern California, United States lgor Delvendahl, University of Zurich, Switzerland Kate O'Connor-Giles, Brown University, United States Rajan Thakur, Brown University,

Providence, United States in collaboration with reviewer $K O^{\prime} \mathrm{C}-\mathrm{G}$

${ }^{*}$ Correspondence: C. Andrew Frank andy-frank@uiowa.edu

${ }^{\dagger}$ Present address: Catherine J. Yeates, Department of Biology, University of Dayton, Dayton, OH, United States

Specialty section: This article was submitted to Cellular Neurophysiology, a section of the journal Frontiers in Cellular Neuroscience

Received: 16 October 2020 Accepted: 13 April 2021 Published: 07 May 2021

Citation: Yeates CJ and Frank CA (2021) Homeostatic Depression Shows Heightened Sensitivity to Synaptic Calcium Front. Cell. Neurosci. 15:618393. doi: 10.3389/fncel.2021.618393

\author{
Catherine J. Yeates ${ }^{1,2 \dagger}$ and C. Andrew Frank ${ }^{1,2,3 *}$ \\ ${ }^{1}$ Department of Anatomy and Cell Biology, University of lowa Carver College of Medicine, lowa City, IA, United States, \\ ${ }^{2}$ Interdisciplinary Graduate Program in Neuroscience, University of lowa, lowa City, IA, United States, ${ }^{3}$ lowa Neuroscience \\ Institute, University of lowa Carver College of Medicine, Iowa City, IA, United States
}

Synapses and circuits rely on homeostatic forms of regulation in order to transmit meaningful information. The Drosophila melanogaster neuromuscular junction (NMJ) is a well-studied synapse that shows robust homeostatic control of function. Most prior studies of homeostatic plasticity at the NMJ have centered on presynaptic homeostatic potentiation (PHP). PHP happens when postsynaptic muscle neurotransmitter receptors are impaired, triggering retrograde signaling that causes an increase in presynaptic neurotransmitter release. As a result, normal levels of evoked excitation are maintained. The counterpart to PHP at the NMJ is presynaptic homeostatic depression (PHD). Overexpression of the Drosophila vesicular glutamate transporter (VGlut) causes an increase in the amplitude of spontaneous events. PHD happens when the synapse responds to the challenge by decreasing quantal content (QC) during evoked neurotransmission-again, resulting in normal levels of postsynaptic excitation. We hypothesized that there may exist a class of molecules that affects both PHP and PHD. Impairment of any such molecule could hurt a synapse's ability to respond to any significant homeostatic challenge. We conducted an electrophysiology-based screen for blocks of PHD. We did not observe a block of PHD in the genetic conditions screened, but we found loss-of-function conditions that led to a substantial deficit in evoked amplitude when combined with VGlut overexpression. The conditions causing this phenotype included a double heterozygous loss-of-function condition for genes encoding the inositol trisphosphate receptor ( $\left(\mathrm{P}_{3} \mathrm{R}-i \mathrm{tpr}\right)$ and ryanodine receptor $(R y R)$. $I_{3} R s$ and RyRs gate calcium release from intracellular stores. Pharmacological agents targeting $\mathrm{IP}_{3} \mathrm{R}$ and RyR recapitulated the genetic losses of these factors, as did lowering calcium levels from other sources. Our data are consistent with the idea that the homeostatic signaling process underlying PHD is especially sensitive to levels of calcium at the presynapse.

Keywords: synapse, homeostasis, depression, Drosophila melanogaster, NMJ, plasticity, neurotransmission 


\section{INTRODUCTION}

Animal nervous systems use forms of homeostatic synaptic plasticity to maintain stable function. Over the last 20-25 years, studies from diverse systems have revealed a wealth of information about how forms of homeostatic synaptic plasticity are implemented (Marder and Goaillard, 2006; Turrigiano, 2008; Pozo and Goda, 2010; Davis, 2013; Davis and Müller, 2015; Delvendahl and Müller, 2019). In particular, work using the Drosophila melanogaster neuromuscular junction (NMJ) has uncovered many facets of homeostatic implementation on a molecular level (Frank, 2014a; Frank et al., 2020). Much of the NMJ homeostasis work in both Drosophila and vertebrates has focused on a form of homeostatic plasticity termed presynaptic homeostatic potentiation (PHP). With PHP, manipulations that impair postsynaptic muscle receptor function trigger an increase in presynaptic vesicle release (Cull-Candy et al., 1980; Petersen et al., 1997; Davis et al., 1998; Frank et al., 2006; Wang et al., 2016).

Homeostatic plasticity at the NMJ is a bi-directional process. First, PHP is reversible-when manipulations that impair muscle receptor function are removed, the presynaptic potentiation ceases (Wang et al., 2016; Yeates et al., 2017). Second, the Drosophila NMJ can depress quantal content (QC) in a homeostatic manner functionally opposite to PHP: presynaptic homeostatic depression (PHD). Experimentally, one way to trigger PHD is to overexpress the Drosophila vesicular glutamate transporter gene, VGlut, in motor neurons. Overexpression of the glutamate transporter leads to an increase in the diameter of glutamatergic vesicles, an increase in quantal size across the entire distribution of spontaneous miniature events, and very large spontaneous quantal events (Daniels et al., 2004). To compensate for this, quantal content at the NMJ is lowered, resulting in normal evoked postsynaptic excitation (Daniels et al., 2004).

Many genes have been shown to be necessary for PHP at the NMJ. But much less is known about PHD. Both PHP and PHD result in opposite changes in quantal content, and studies suggest divergent and separable mechanisms governing these forms of homeostatic plasticity. Some genes required for homeostatic potentiation are dispensable for homeostatic depression (Marie et al., 2010; Gaviño et al., 2015; Li et al., 2018). Moreover, unlike homeostatic potentiation, homeostatic depression does not appear to involve a change in the size of the readily releasable pool of synaptic vesicles (Li et al., 2018). Rather, homeostatic depression appears to involve a decrease in release probability (Gaviño et al., 2015). Finally, PHP at the NMJ appears to be a process that is dependent on the input (i.e., the type of synapse formed at the NMJ; Newman et al., 2017) while PHD does not appear to be input specific (Li et al., 2018).

The degree of overlap between homeostatic depression and homeostatic potentiation is unknown. We designed a smallscale, directed screen to test for links between these two forms of homeostatic plasticity. For the screen, we targeted genes based on prior evidence that their impairment in the neuron caused a failure of the long-term maintenance of PHP.
We examined loss-of-function conditions for these genes in a VGlut overexpression background for PHD. We did not find any cases of failed homeostatic depression - the conditions we examined showed decreases in quantal content in response to increased quantal size. However, we found an interesting and unexpected evoked neurotransmission phenotype: a robust decrease in excitatory postsynaptic potential (EPSP) amplitude in a VGlut-overexpressing genetic background. We observed this phenotype for a double heterozygous loss-of-function condition for the Ryanodine and $\mathrm{IP}_{3}$ receptor-encoding genes. In our follow-up work, pharmacology phenocopied this genetic result, and our overall findings are consistent with the idea that the PHD system may show a heightened sensitivity to low calcium.

Our findings highlight a novel synaptic transmission phenotype. Prior characterizations of homeostatic depression do not report decreases in EPSP amplitude in VGlut overexpression relative to controls (Daniels et al., 2004; Marie et al., 2010; Gaviño et al., 2015; Li et al., 2018). Studies at the NMJ have resulted in models in which homeostatic compensation maintains evoked neurotransmission at the synapse approximately at control levels (Davis, 2013). Our results suggest that impairing store calcium channels may result in a cumulative defect in neurotransmission when there is a concurrent PHD challenge. We find this interesting, especially in light of the fact that these same store channels are required for the maintenance of PHP (James et al., 2019) and because other recent studies in other systems have implicated store calcium in presynaptic release mechanisms (e.g., de Juan-Sanz et al., 2017).

\section{MATERIALS AND METHODS}

\section{Drosophila Stocks and Husbandry}

Fruit fly stocks were obtained from the Bloomington Drosophila Stock Center (BDSC, Bloomington, Indiana), Kyoto Stock Center (DGRC, Kyoto, Japan), Japan National Institute of Genetics (Mishima, Shizuoka, Japan), Vienna Drosophila Research Center (VDRC, Vienna, Austria), or from the labs that generated them. $w^{1118}$ was used as a wild-type (WT) control (Hazelrigg et al., 1984). RNAi lines and mutants used in the screen are reported in Supplementary Table 1.

Fruit flies were raised on cornmeal, molasses, and yeast medium (see BDSC website for standard recipe) in temperaturecontrolled conditions. Animals were reared at $25^{\circ} \mathrm{C}$ until they reached the wandering third instar larval stage, at which point they were selected for electrophysiological recording. UAS-VGlut (Daniels et al., 2004) was recombined with OK371-GAL4 (Mahr and Aberle, 2006; Meyer and Aberle, 2006) to drive constitutive overexpression of VGlut. The full genotype of these animals is: $w$; VGlut, OK371-Gal4/CyO-GFP. Virgins of these flies were crossed to RNAi lines or mutants to test for changes to homeostatic depression. w; OK371-Gal4/+ was used as a genetic control for baseline electrophysiology.

\section{Electrophysiology and Analysis}

Larvae were dissected in a modified HL3 saline comprised of: $\mathrm{NaCl}(70 \mathrm{mM}), \mathrm{KCl}(5 \mathrm{mM}), \mathrm{MgCl}_{2}(10 \mathrm{mM}), \mathrm{NaHCO}_{3}$ 
(10 $\mathrm{mM})$, sucrose $(115 \mathrm{mM}=3.9 \%)$, trehalose $(4.2 \mathrm{mM}=0.16 \%)$, HEPES (5.0 $\mathrm{mM}=0.12 \%)$, and $\mathrm{CaCl}_{2}(0.5 \mathrm{mM}$, except as noted).

For pharmacology, Dantrolene (R\&D Systems) and Xestospongin C (Abcam) were used. Dantrolene was mixed into saline to a final concentration of $25 \mu \mathrm{M}$. Larvae were cut open on the dorsal side and allowed to incubate in the Dantrolene saline for $5 \mathrm{~min}$. The rest of the dissection and recording was completed in Dantrolene saline. Xestospongin C was applied in a similar manner, with the animals allowed to incubate in $20 \mu \mathrm{M}$ Xestospongin $\mathrm{C}$ saline for $5 \mathrm{~min}$ before they were recorded, also in saline containing Xestospongin C.

Electrophysiological data were collected using an Axopatch 200B amplifier (Molecular Devices, Sunnyvale, CA, USA) in bridge mode, digitized using a Digidata 1440A data acquisition system (Molecular Devices), and recorded with pCLAMP 10 acquisition software (Molecular Devices). A Master-8 pulse stimulator (A.M.P. Instruments, Jerusalem, Israel) and an ISO-Flex isolation unit (A.M.P. Instruments) were utilized to deliver $1 \mathrm{~ms}$ suprathreshold stimuli to the appropriate segmental nerve. The average spontaneous miniature excitatory postsynaptic potential (mEPSP) amplitude per NMJ was quantified by hand, approximately 100 individual spontaneous release events per NMJ (MiniAnalysis, Synaptosoft, Fort Lee, NJ, USA). Measurements from all NMJs of a given condition were then averaged. For evoked neurotransmission, 30 excitatory postsynaptic potentials (EPSPs) were averaged to find a value for each NMJ. These were then averaged to calculate a value for each condition. QC was calculated by the ratio of average EPSP and average mEPSP amplitudes for each individual NMJ. An average quantal content was then calculated for each condition. EPSP variability was assessed by measuring each of the 30 traces individually and calculating a standard deviation, coefficient of variation, and range for that NMJ. Range was defined as the maximum EPSP value minus the minimum EPSP value.

\section{Immunostaining}

An immunostaining experiment is detailed in Figure 4. Procedures match those previously published (Brusich et al., 2015, 2018; Spring et al., 2016; Yeates et al., 2017; James et al., 2019). Briefly, third instar larvae were filleted and fixed for 5 min with Bouin's fixative (Ricca Chemical, Arlington, TX, USA). After washes, fixed fillets were incubated in primary antibodies overnight at $4^{\circ} \mathrm{C}$, mouse anti-Brp (nc82, 1:250, University of Iowa Developmental Studies Hybridoma Bank; Wagh et al., 2006) and rabbit anti-Dlg (1:5,000; Budnik et al., 1996). After washes, fillets were incubated in fluorophoreconjugated secondary antibodies overnight at $4^{\circ} \mathrm{C}$ (Jackson ImmunoResearch Labs, West Grove, PA, USA), goat antimouse-488 (DyLight, 1:1,000) and goat anti-rabbit-549 (DyLight, 1:2,000). After washes, fillets were mounted and Dlg boutons were counted blinded by hand on an epifluorescence microscope and double checked for Brp signal in apposition. Note: relative bouton numbers between NMJs 6/7 on segment A2 and A3 are consistent with earlier studies, though some raw numbers appear slightly lower, which may be due either to hand counting (rather than automated) or due to Dlg signal bouton counting (rather than HRP signal counting).

\section{Statistical Analyses}

Statistical analyses were conducted using GraphPad Prism software. Statistical significance was assessed either by Student's $t$-test when one experimental dataset was being directly compared to a control dataset, or one-way ANOVA with Tukey's post-hoc test when multiple datasets were being compared. For Figure 5, statistical tests were run as a two-way ANOVA with Tukey's post-hoc to test the effects of both genotype and Dantrolene application. Specific $p$-value ranges are noted in the Figure legends and shown in graphs as follows: ${ }^{\star} p<0.05$, ${ }^{* *} p<0.01$, and ${ }^{* * *} p<0.001$ ( $^{*}$ and ${ }^{*}$ are used in Figures if there are additional comparisons highlighted). For some comparisons that are close to $p<0.05$ statistical significance but do not achieve it $(0.05<p<0.1)$, specific values are reported on the graph itself. Calcium cooperativity data were analyzed using a non-linear fit regression analysis on GraphPad Prism.

\section{RESULTS}

\section{A Recombinant Line to Analyze Presynaptic Homeostatic Depression (PHD)}

Using previously published reagents, we generated a fly stock with constitutive VGlut transgene overexpression. Such a stock could be used as a tool for a single-cross genetic screen. To generate the stock, we recombined the OK371-Gal4 motor neuron driver (Mahr and Aberle, 2006; Meyer and Aberle, 2006) with a UAS-VGlut transgene (Daniels et al., 2004). We placed these two genetic elements in cis on Drosophila melanogaster Chromosome II. OK371-Gal4 is an enhancer trap line for the VGlut promoter itself. This ensured that GAL4-driven UAS-VGlut overexpression would happen in desired tissues, Drosophila motor neurons.

We tested if the recombinant line constitutively overexpressing UAS-VGlut could express PHD at the NMJ. We crossed the recombinant stock to our wild-type stock ( $w^{1118}$, herein: WT; Cross result, herein: "VGlut, OK371/+"). By NMJ electrophysiology, we recorded from WT control, OK371/+ control, and w; VGlut, OK371/+. As expected, VGlut, OK371/+ NMJs showed an increase in spontaneous miniature excitatory postsynaptic potential (mEPSP) amplitude compared to controls (Figures 1A-C; data also in Supplementary Table 1). Compared to WT control NMJs, there was no significant difference in evoked postsynaptic amplitudes for VGlut, OK371/+ NMJs (Figure 1D; $p=0.82$, one-way ANOVA). This was because of an accurate homeostatic decrease in QC (Figure 1E)-hence, successful PHD. This result matched earlier studies that had used WT as a control and a trans OK371/UAS-VGlut combination to induce PHD (Daniels et al., 2004; Gaviño et al., 2015; Li et al., 2018).

Even though PHD was successful relative to WT for our test cross, we noted a small, but statistically significant, baseline increase in the EPSP amplitude of OK371/+ NMJs. This increase in $O K 371 /+$ EPSP level was present compared either to WT control or to VGlut, OK371/+ (Figure 1D). One possibility is that the $O K 371 /+$ genetic background has slightly elevated 
A

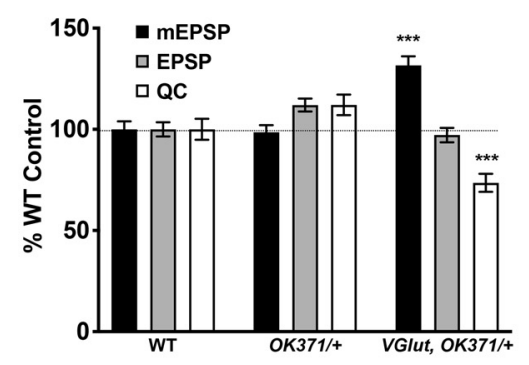

B

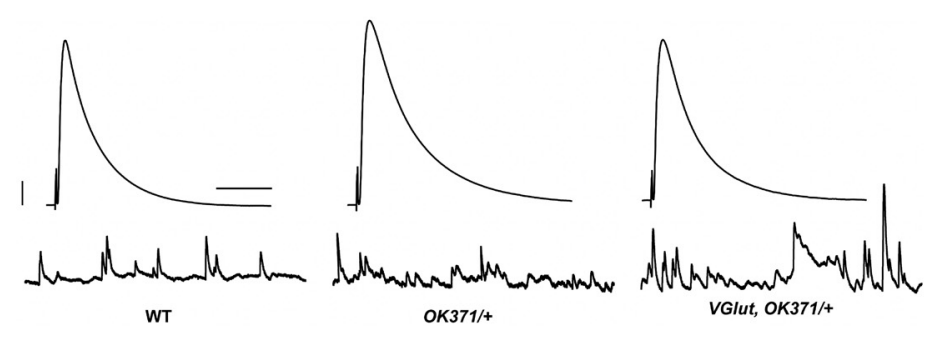

C

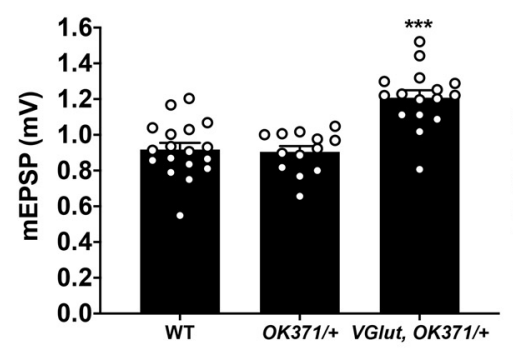

E

D

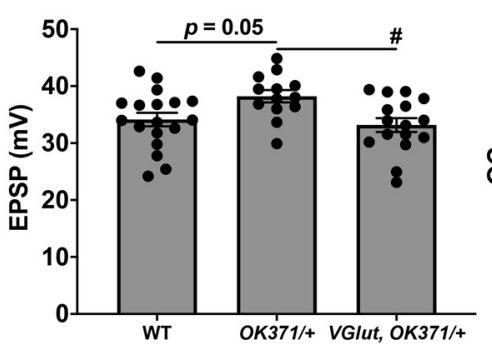

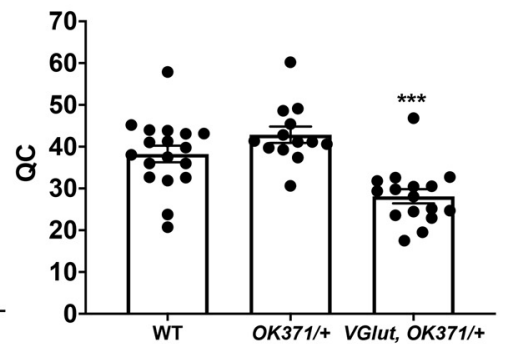

FIGURE 1 | Presynaptic homeostatic depression (PHD) works successfully with a recombinant line of OK371-Ga/4 and UAS-VG/ut. (A) Neuromuscular junction (NMJ) electrophysiological data for miniature excitatory postsynaptic potentials (mEPSP), excitatory postsynaptic potentials (EPSP), and quantal content (QC). Data are normalized to wild-type (WT; $w^{1118}$ ) values. VG/ut, OK371/+ NMJs have increased mEPSP but normal EPSP because of decreased QC, indicative of successful PHD ( ${ }^{* * *} p<0.001$ vs. WT by one-way ANOVA with Tukey's post-hoc). (B) Representative electrophysiological traces. Large traces are EPSPs; small traces are mEPSPs. Scale bars for EPSPs (mEPSPs) are $5 \mathrm{mV}(1 \mathrm{mV})$ and $50 \mathrm{~ms}(1,000 \mathrm{~ms})$. (C) Raw data for mEPSPs. (D) Raw data for EPSPs. (E) Raw data for QC. For (C-E), bars are averages and error bars are \pm SEM. ${ }^{* \star *} p<0.001$ vs. WT or vs. OK371/+; ${ }^{*} p<0.05$ vs. OK371/+; analyses by one-way ANOVA with Tukey's post-hoc.

release, and the combined addition of UAS-VGlut reveals a slight depression in evoked amplitude. Noting this potentially important difference in our driver control, we continued using the $O K 371 /+$ heterozygous condition as a genetic background control. OK371/+ is a closer genetic control for PHD analysis than WT.

\section{A Genetic Screen Identifies an Interaction Between Calcium Stores and a PHD-Inducing Challenge}

We used our recombinant line to conduct a genetic screen for conditions that affect PHD. We crossed this stock to screen stocks: (1) either to drive UAS-RNAi transgenes to knock down genes; (2) to drive other chosen UAS transgenes; or (3) to combine with heterozygous loss-of-function mutant lines ("Materials and Methods" section, Figure 2A). For the screen, we targeted a subset of genes previously identified as in the neuron for homeostatic potentiation, or closely related genes. We tested 43 genotypes (sometimes multiple conditions for a single gene), including our homeostatic depression condition, VGlut, OK371/+ (Figures 2B,C).

The aggregate results of the screen are reported here (Figures 2B,C; raw data in Supplementary Table 1). We recorded from 42 experimental heterozygous mutant/+ or $>U A S$ RNAi or UAS-transgene/+ conditions, in the VGlut, OK371/+ genetic background. Of those 42, 12 achieved EPSPs that were numerically larger than VGlut, OK371/+, and 22 achieved QCs that were numerically larger than VGlut, OK371/+ (Figures 2B,C). Increased evoked potentials could signify failed PHD-however, none of these cases represented statistically significant increases compared to VGlut, OK371/+. None were so much bigger that they were good candidates for "failed PHD." Indeed, all of the candidates had average EPSP and QC levels below OK371/+ NMJ baseline recordings (compare Figures 1D,E, 2B,C).

We recognize limitations in this kind of screening analysis. For example, we expect a certain degree of negatives or false negatives for any screen. In our case, there could be false negatives due to a limited scope of examination, the effects of non-linear summation by measuring large synaptic voltages, or due to varying baseline parameters from genotype to genotype (Supplementary Table 1).

Despite the negative results, we noted a phenotype distinct from what we were initially seeking: two crosses yielded larvae with striking decreases in NMJ EPSP amplitudes, more than two standard deviations below the average EPSPs from the baseline VGlut, OK371/+ dataset (Figure 2B). One case was knockdown of the Survival motor neuron (Smn) gene with the UAS-Smn[RNAi] $]^{J 02057}$ line in the VGlut, OK371/+ background. This was intriguing because Drosophila Smn is homologous to human SMN. Defects in SMN cause Spinal Muscular Atrophy (Lefebvre et al., 1995). Drosophila Smn has been characterized 
A

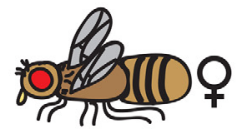

w; UAS-VGlut, OK371-Gal4 X mut OR UAS-gene[RNAi]

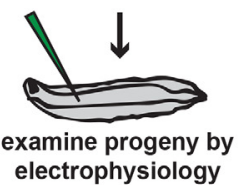

C
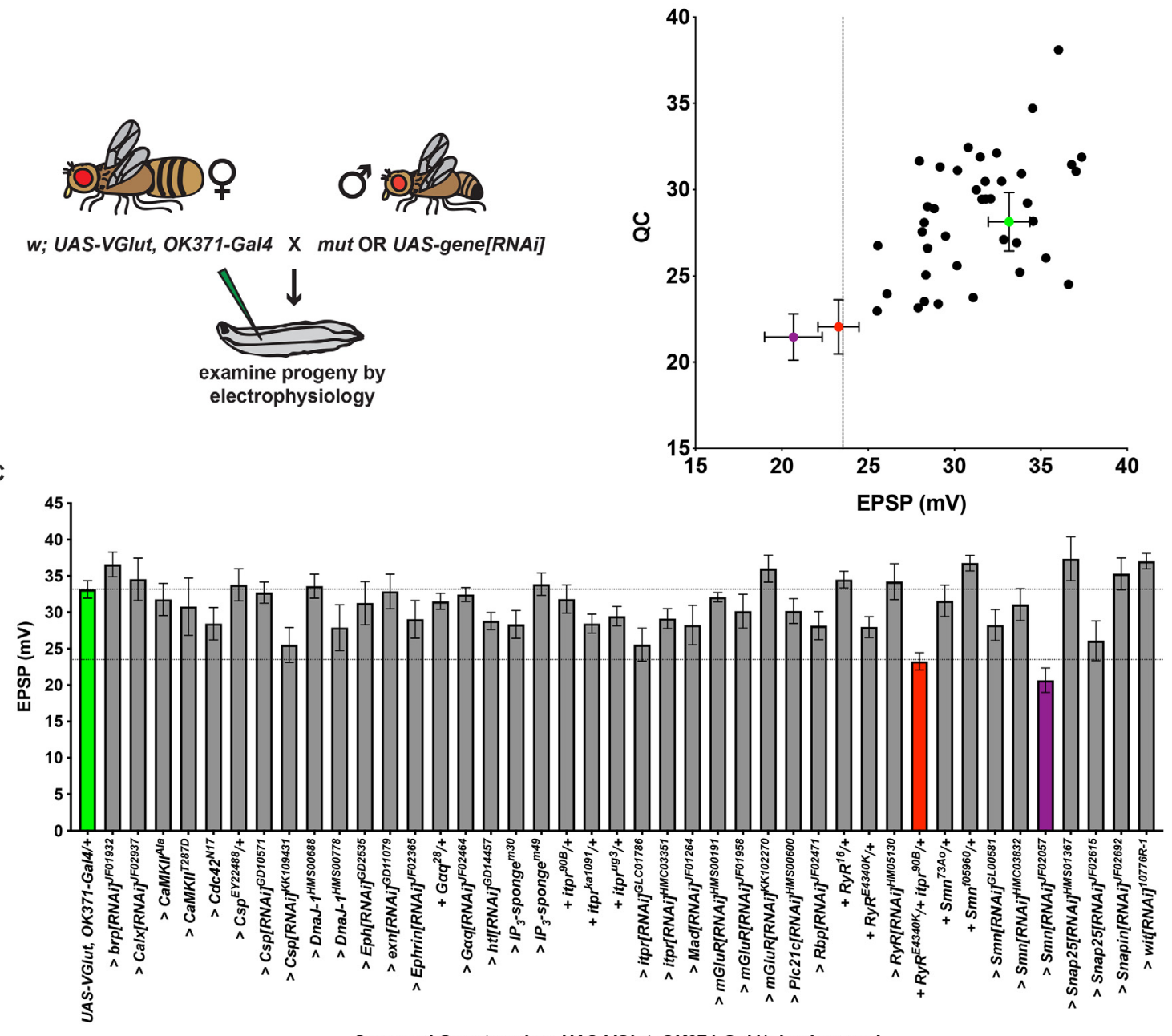

Screened Genotype in a UAS-VG/ut, OK371-Gal4/+ background

FIGURE 2 | An electrophysiology screen in a PHD-challenged genetic background. (A) Crossing scheme for generating larvae for electrophysiological recording. Each animal recorded had a homeostatic challenge provided by VGlut overexpression and a concurrent heterozygous or RNAi condition. Fly artwork reproduced from Brusich et al. (2015) under a Creative Commons Attribution License. (B) Data distribution for screened conditions (X-axis = average EPSP for condition; y-axis = average QC for condition). Green = UAS-VG/ut, OK371-Ga/4/+. Red = UAS-VG/ut, OK371-Gal4/RyR E4340K; itpr ${ }^{90}$ B/+. Purple = UAS-VG/ut, OK371-Gal4/+; UAS-Smn[RNAi]fF2057/+. Dotted line: EPSP value two standard deviations below UAS-VG/ut, OK371-Ga/4/+ chosen as a cut off for potential follow-up hits. (C) Average EPSPs for screened conditions. All conditions have a UAS-VG/ut, OK371-Gal4/+genetic background. ">" denotes as UAS construct or RNAi line being driven in motor neurons by OK371-Gal4. "+" denotes additional mutations present as heterozygotes. Top dotted line denotes UAS-VG/ut, OK371-Ga/4/+ average. Bottom dotted line denotes two standard deviations below UAS-VG/ut, OK371-Ga/4/+ average.

as a potential model for Spinal Muscular Atrophy (Sen et al., 2011; Spring et al., 2019; Raimer et al., 2020). Smn has also previously been implicated in PHP (Sen et al., 2011). However, the result for UAS-Smn[RNAi] JF02057 was not replicated by other Smn knockdown or loss-of-function mutant test crosses (Figures 2B,C).We did not follow up on Smn for this study.

A second case with a striking decrease in EPSP amplitude in the screen was a double heterozygous genetic condition in genes encoding the Drosophila Ryanodine receptor $(R y R)$ and inositol 1,4,5-trisphosphate $\left(\mathrm{IP}_{3}\right)$ receptor (itpr): VGlut, OK371/RyR $R^{E 4340 K} ; \quad i t p r^{90 B} /+$ (Figures 2B,C). Ryanodine receptors (RyRs) and $\mathrm{IP}_{3}$ receptors $\left(\mathrm{IP}_{3} \mathrm{Rs}\right)$ are localized to the endoplasmic reticulum. They mediate release of calcium from intracellular stores (Berridge, 1984, 1987, 1998; Simkus and Stricker, 2002). The $R y R^{E 4340 K}$ mutation is a single amino acid substitution (glutamic acid to lysine; Dockendorff et al., 2000), and the $\operatorname{ttpr}^{90 B}$ mutation is a null mutant generated by imprecise excision of a transposon (Venkatesh and Hasan, 1997). We previously defined roles for $\mathrm{RyR}, \mathrm{IP}_{3} \mathrm{R}, \mathrm{IP}_{3}$ signaling and upstream components in maintaining PHP (Brusich et al., 2015; James et al., 2019).

In parallel, we screened single mutant manipulations for both genes. Neither the $R y R^{E 4340 K} /+$ heterozygous condition, nor the $\operatorname{itpr}^{90 B} /+$ heterozygous condition-nor any single heterozygous 

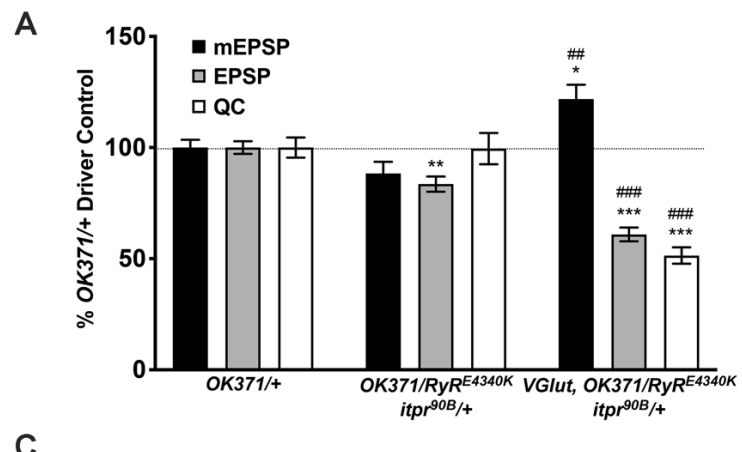

C

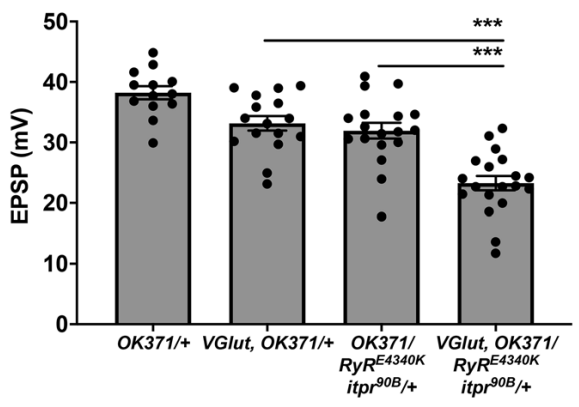

$E$

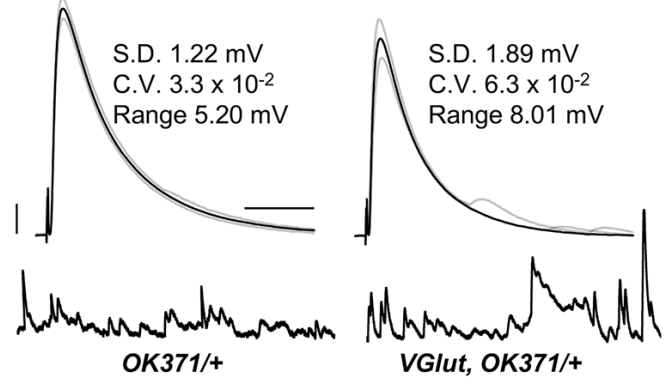

B

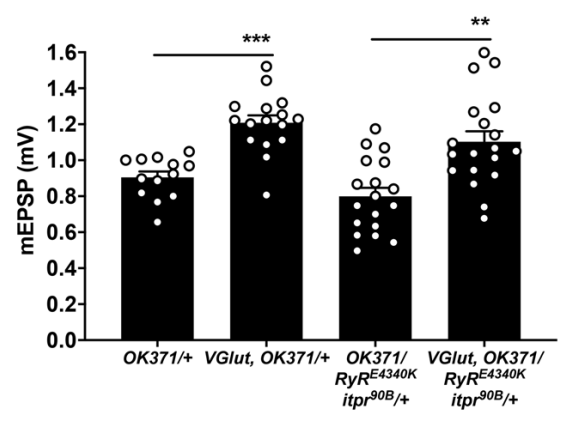

D

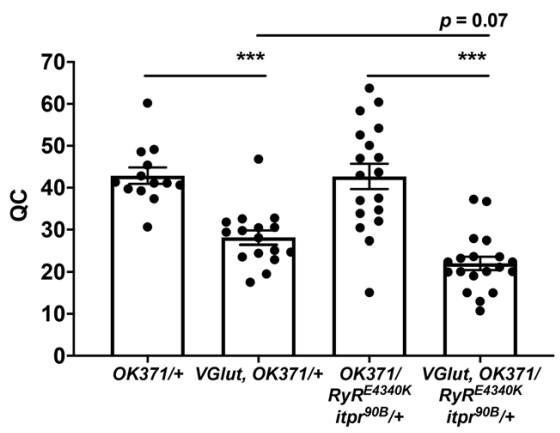

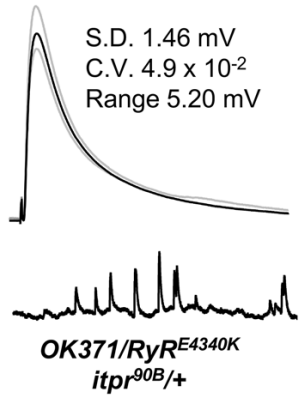

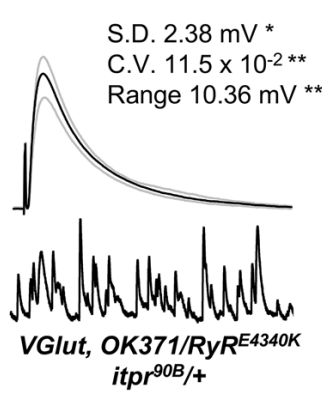

FIGURE 3 | Double heterozygous loss of the itpr and RyR genes interacts with the PHD challenge to diminish neurotransmission. Note: traces and data for OK371/+ and VG/ut, OK371/+ are repeated from Figure 1, for genetic background comparison. Abbreviations are as in Figure 1. (A) NMJ electrophysiological data

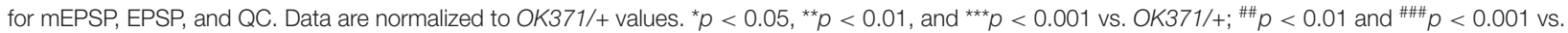
OK371/RyR ${ }^{E 4340 K}$; itpr 90 B /+; analyses by one-way ANOVA with Tukey's post-hoc. (B) Raw data for mEPSPs. (C) Raw data for EPSPs. (D) Raw data for QC. For (B-D), bars are averages and error bars are \pm SEM. ${ }^{*} p<0.05,{ }^{* *} p<0.01$, and ${ }^{* * *} p<0.001$ by one-way ANOVA across genotypes, with Tukey's post-hoc. (E) Representative electrophysiological traces with standard deviation (SD), coefficient of variation (CV) and range values for EPSPS. The SD, CV, and range were significantly higher for VG/ut, OK371/Ry $R^{E 4340 K}$; itpr $r^{0 \mathrm{OB}} /+$ vs. its genetic control, OK371/Ry $R^{E 4340 K}$; itpr $r^{0 \mathrm{~B}} /+.{ }^{*} p<0.05,{ }^{* *} p<0.01$ by one-way ANOVA across genotypes, with Tukey's post-hoc. Scale bars as in Figure 1.

or RNAi knockdown condition for either gene-yielded as significantly depressed EPSPs in response to PHD challenge (Figures 2B,C). Therefore, the screen result with the double heterozygote could be due to a genetic interaction, or it could be due to other factors in the genetic background. This preliminary finding required further characterization.

We tested if the electrophysiological phenotype could be due to a baseline neurotransmission defect when both genes are heterozygous. By electrophysiology, we compared NMJs from $O K 371 / R y R^{E 4340 K} ; i t p r^{90 B} /+$ larvae as a baseline double heterozygous condition vs. NMJs from VGlut, OK371/Ry $R^{E 4340 K}$; $i_{\text {tpr }} \mathrm{r}^{9 \mathrm{~B}} /+$ larvae (Figures 3A-D). Just like WT, the baseline double heterozygous condition did have a slight decrease in EPSP amplitude compared to OK371/+ driver control (Figure 3A). This indicated a small, but discernible defect in neurotransmission in animals where the $\mathrm{IP}_{3} \mathrm{Rs}$ and RyRs are both impaired. The double heterozygous condition with concurrent VGlut gene overexpression showed a further decrease in transmission-compared to its own genetic control, it had increased quantal size (Figure 3B), but significantly decreased evoked amplitude (Figure 3C) because of a large decrease in quantal content (Figure 3D). Finally, the quantal content for VGlut, OK371/RyR $R^{E 4340 K}$; itpr ${ }^{90 B} /+$ NMJs was numerically smaller than for VGlut, OK371/+ NMJs (Figure 3D), but this latter numerical difference was not statistically significant $(p=0.07$, one-way ANOVA). 
We noted that the EPSP amplitude in individual VGlut, OK371/RyR $R^{E 4340 K} ; i t p r^{90 B} /+$ NMJ recordings varied markedly from stimulus to stimulus. High variability could indicate unstable neuronal excitability or release. To check if evoked release events were indeed more variable, we completed additional analyses. First, we extracted the amplitude of each individual EPSP event at every NMJ recorded. From these data, we calculated the EPSP standard deviation (SD) and coefficient of variation (CV) per individual NMJ. We also calculated a range for each NMJ by subtracting the maximum EPSP measured at each $\mathrm{NMJ}$ from the minimum. We averaged these $\mathrm{SD}, \mathrm{CV}$, and range measures for each genotype, considering all of the individual EPSP recordings. For all of these EPSP parameters, w; VGlut, OK371/RyR $R^{E 4340}$; $i t r^{90 B} /+$ animals showed statistically significant higher variability compared to controls (Figure 3E). By contrast, double heterozygous baseline OK371/RyR $R^{E 4340 K}$; itpr $r^{90 B} /+$ NMJs did not differ significantly from $w$; OK371/+ driver control NMJs ( $p>0.85$ for each measure, Kruskal-Wallis ANOVA), suggesting that the variability stems from VGlut overexpression in the mutant background (Figure 3E). w; VGlut, OK371/+ NMJs showed numerically higher variability than $w$; OK371/+, but this was not statistically significant (Figure 3E, $p>0.25$ for each measure, Kruskal-Wallis ANOVA).

Finally, we conducted immunostaining to check if any of these electrophysiological defects might correspond with defects in synaptic growth. We assessed growth by co-staining with antibodies against the postsynaptic PSD-95 homolog, DLG (Budnik et al., 1996) and the presynaptic active zone protein, BRP (Wagh et al., 2006). We counted boutons encased by anti-DLG signal and checked that these boutons were apposed by anti-BRP signal. By this analysis, we saw no significant changes in NMJ growth: neither the PHD challenge; nor the double heterozygous loss of the $R y R /+$ and itpr/+ genes; nor combining those manipulations together yielded significant numerical differences in bouton count ( $p>0.90$ for all comparisons, one-way ANOVA; Figures $4 \mathbf{A}, \mathbf{B})$. One caveat to these results is that we only examined these NMJs at the level of bouton count, not at the level of the abundance of specific active zone markers (as in Böhme et al., 2019; Goel et al., 2019; Gratz et al., 2019).

\section{Pharmacology Targeting Ryanodine and $\mathrm{IP}_{3}$ Receptors Recapitulates Loss-of-Function Genetics}

We tested if the electrophysiological phenotypes we observed could be recapitulated by combining genetics and pharmacology. We started with the drug Dantrolene. Dantrolene is a RyR antagonist (Zhao et al., 2001; Vazquez-Martinez et al., 2003). In earlier work at the Drosophila NMJ, we found that application of Dantrolene can abrogate the long-term maintenance of PHP (James et al., 2019).

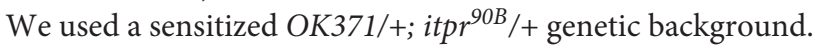
With this background, we could pharmacologically impair RyRs while also genetically impairing $\mathrm{IP}_{3}$ Rs. We applied 25 MM Dantrolene to: (1) OK371/+ NMJs; (2) VGlut, OK371/+ NMJs; (3) OK371/+; itpr ${ }^{90 B /+}$ NMJs; and (4) VGlut, OK371/+; $i \mathrm{ppr}^{90 \mathrm{~B} /}+\mathrm{NMJs}$. We also compared these conditions to a set of data for genetically identical conditions without drug treatment (Figures 5A-C). With two-way ANOVA statistical analyses for our electrophysiological measures, we were able to account separately for genotype effects and Dantrolene effects or interactions between the two.

In the absence of drug treatment, PHD proceeded normally (Figures 5A-C). We noted that the untreated OK371/+; itpr $r^{90 B} /+$ heterozygous condition had a slightly diminished evoked amplitude compared to OK371/+ (Figure 5B). Therefore, the $i \mathrm{tpr}^{90 \mathrm{~B} /+}$ condition could be contributing some neurotransmission loss on its own. But the addition of VGlut transgenic expression to this heterozygous background did not further decrease evoked neurotransmission (Figure 5B), indicating normal PHD, as signified by an expected decrease in quantal content (Figure 5C).

With $25 \mu \mathrm{M}$ Dantrolene treatment, the data were more complex. First, mEPSP amplitudes were generally smaller than
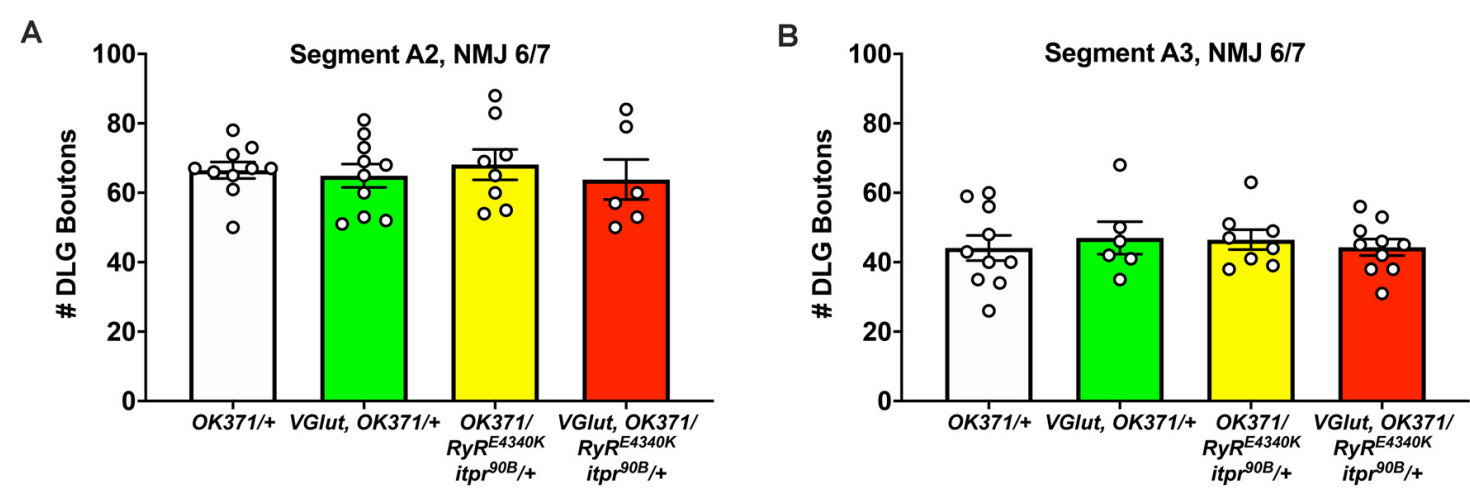

FIGURE 4 | No discernible NMJ growth defects. NMJs of third instar larvae (same genotypes as Figure 3) were analyzed by immunostaining, co-staining with anti-DLG for the postsynaptic density and anti-Brp to check for apposed presynaptic active zones. (A) DLG boutons counted for Segment A2, NMJ 6/7. (B) DLG boutons counted for Segment A3, NMJ 6/7. No significant differences were found across genotypes ( $p>0.9$ for every possible head-to-head comparison, one-way ANOVA). 
without treatment (Figure 5A). Paradoxically, such a decrease could potentially trigger a short-term induction of PHP in the baseline $O K 371 /+$ condition (Figure 5C) - even though a lower dose of Dantrolene actually abrogates the long-term maintenance of PHP (James et al., 2019). Yet even if this is the case, we are able to do our analysis. A prior study demonstrated that the PHP and PHD processes can occur additively at the same NMJ without interference (Li et al., 2018).

For our experiments with Dantrolene in the VGlutoverexpressing backgrounds, mEPSPs were elevated compared to their respective genetic controls with Dantrolene (Figure 5A). This indicated that in the presence of Dantrolene, VGlut overexpression still caused homeostatic pressure that could induce PHD. Additionally, with Dantrolene, EPSP amplitudes in VGlut-overexpressing lines were decreased compared to their respective genetic controls (Figures 5B). This was due to large decreases in quantal content (Figure 5C).

Interestingly, the VGlut, OK371/+; itpr $r^{90 B} /+$ condition (+Dantrolene) had depressed evoked amplitudes compared either to the VGlut, OK371/+ (+Dantrolene) condition $(p<0.01$, two-way ANOVA) or to the OK371/+; itpr $r^{90 B} /+$ (+Dantrolene) condition ( $p=0.07$, two-way ANOVA; Figure 5B). We note that the latter case does not achieve statistical significance on its own. However, two-way ANOVA analyses on the datasets show that both EPSP amplitudes and quantal content have a significant degree of their variation explained by an interaction between genotype and Dantrolene application (Figure 5D).

Collectively, our data could indicate a cumulative neurotransmission defect when impairing both the $\mathrm{IP}_{3} \mathrm{Rs}$ and RyRs in a PHD-challenged background (electrophysiological traces, Figure 5E). We needed to test this idea further with more combinations and genetic conditions.

It is possible that strong impairment of RyRs could be sufficient to cause synthetic phenotypes in conjunction with the PHD regulation system. We reasoned that Dantrolene might be able to exert strong effects in a heterozygous $R y R /+$ background because this is not a null $R y R$ genetic condition. Therefore, we ran additional pharmaco-genetic tests using a second sensitized genetic background, OK371/RyR $R^{E 4340 K}$ —both with and without drugs and with and without UAS-VGlut overexpression. Again, in the absence of pharmacological treatment, PHD proceeded normally in the heterozygous
A

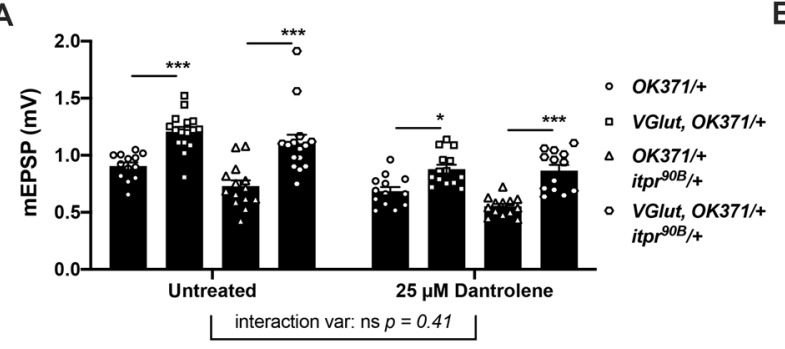

C

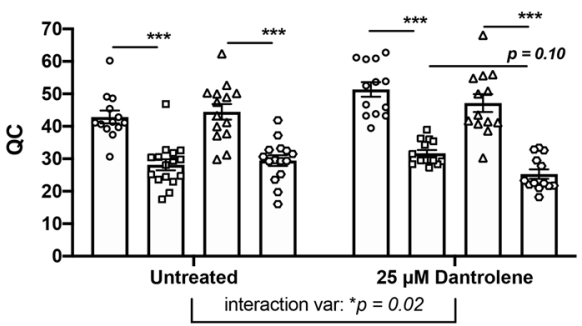

B

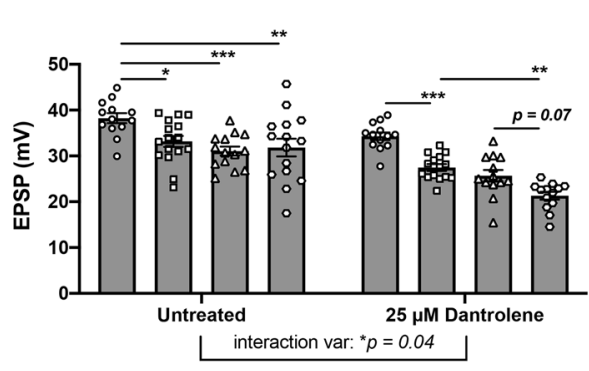

D Two-Way ANOVA Sources of Data Variation

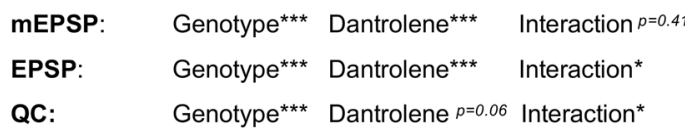

E

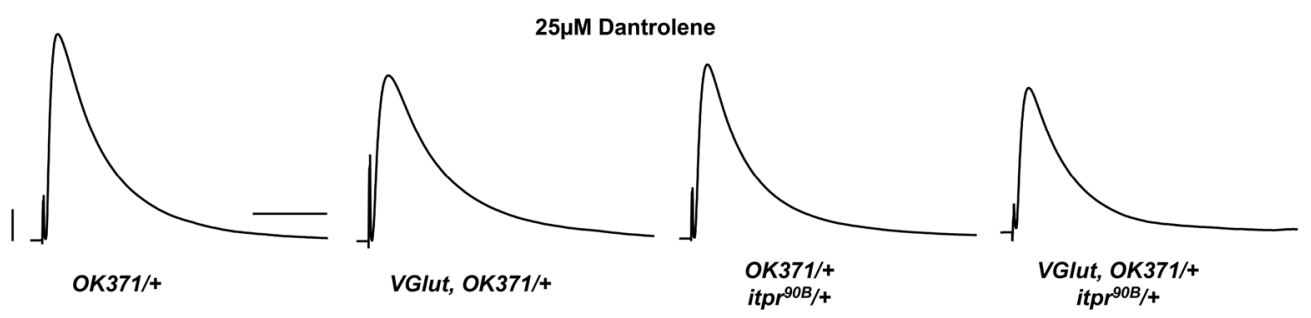

FIGURE 5 | Genetic impairment of itpr combined with pharmacological impairment of RyR phenocopies genetic findings. Notes: Untreated data for OK371/+ and VG/ut, OK371/+ are repeated from Figure 1, for genetic background comparison, and analyses are by two-way ANOVA with Tukey's post-hoc to account for genotype effects, $25 \mu \mathrm{M}$ Dantrolene effects, and interaction effects between genotype and Dantrolene. (A) Raw data for mEPSPs. (B) Raw data for EPSPs. (C) Raw data for QC. For (A-C), bars are averages and error bars are \pm SEM, with individual datapoint shapes corresponding to genotype. (D) Two-way ANOVA analysis of the effects of each parameter on data variation for each electrophysiological measure. ${ }^{*} p<0.05,{ }^{* *} p<0.01$, and ${ }^{* * *} p<0.001$ by two-way ANOVA with Tukey's post-hoc. (E) Representative EPSP traces. Scale bars are as in Figure $\mathbf{1}$. 
A

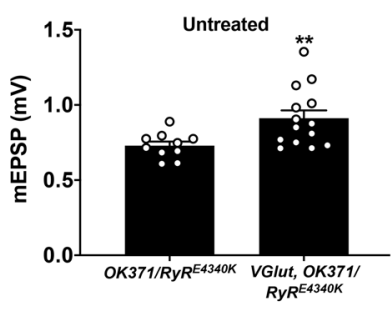

B

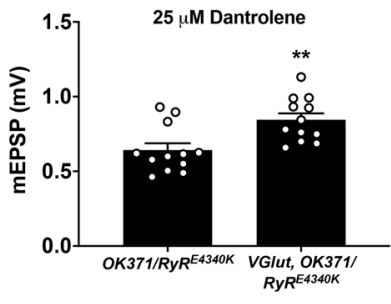

C

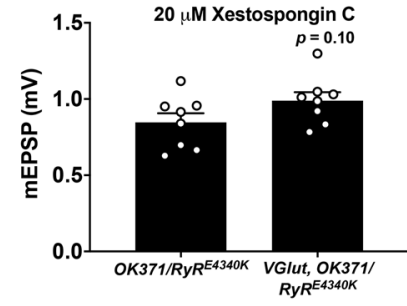

D

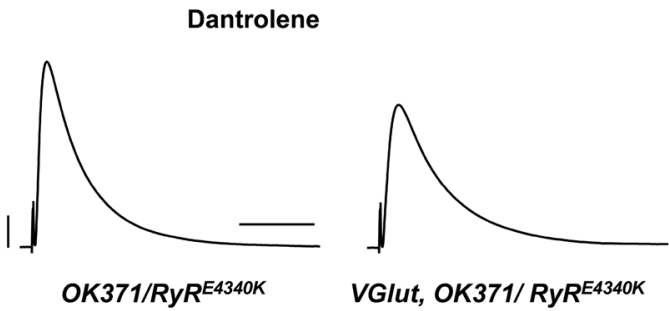

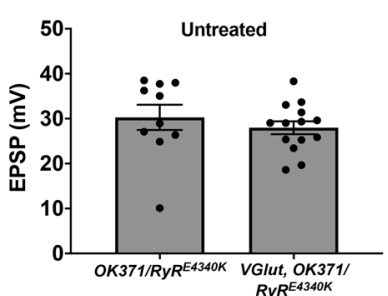
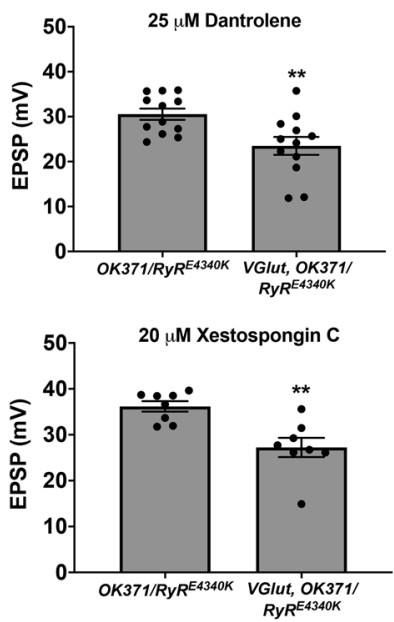
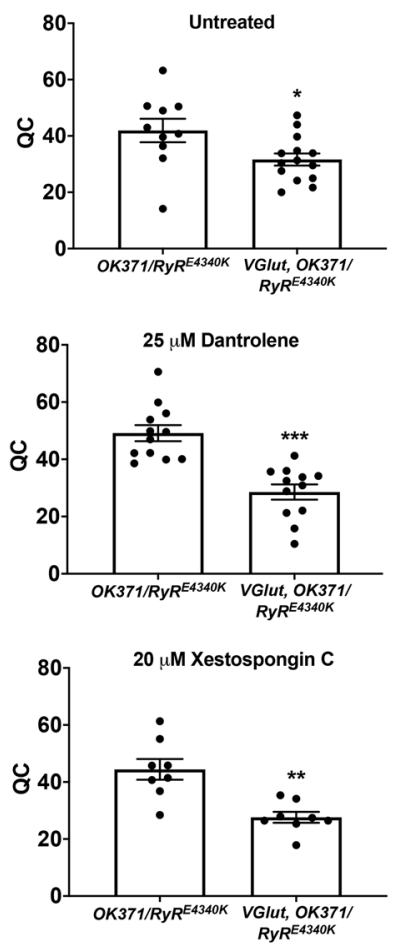
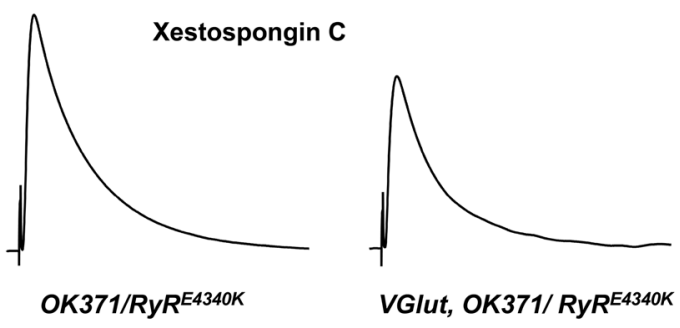

FIGURE 6 | Additional pharmaco-genetic combinations phenocopy the genetic conditions. (A) Raw data for mEPSPs (left); raw data for EPSPs (middle); raw data for QC (right) for untreated genotypes as shown; bars are averages and error bars are \pm SEM. (B) Data as in (A) but with $25 \mu \mathrm{M}$ Dantrolene added to NMJ preps. (C) Data as in (A) but with $20 \mu \mathrm{M}$ Xestospongin C added to NMJ preps. (D) Representative EPSP traces. Scale bars are as in Figure 1. ${ }^{*} p<0.05,{ }^{* *} p<0.01$, and ${ }^{* * *} p<0.001$ by Student's $t$-test comparing a control dataset (no VGlut overexpression) vs. an experimental dataset (VGlut overexpression).

OK371/RyR E4340K genetic background (Figure 6A). With Dantrolene, mEPSPs became significantly larger when VGlut was expressed (Figure 6B, left), but EPSPs were significantly reduced (Figures 6B, middle, 6D) because of a decrease in quantal content (Figure 6B, right).

Finally, we attempted the inverse pharmaco-genetic experiment from that in Figure 5. This time we used the $\mathrm{IP}_{3} \mathrm{R}$ inhibitor, Xestospongin C (Gafni et al., 1997; Wilcox et al., 1998) and the sensitized $O K 371 / R y R^{E 4340 K}$ genetic background. We applied $20 \mu \mathrm{M}$ Xestospongin C, both to $O K 371 / R y R^{E 4340 K} \mathrm{NMJs}$ and to VGlut, OK371/RyR ${ }^{E 4340 K}$ NMJs. mEPSPs were numerically larger when VGlut was overexpressed (Figure 6C, left)-though interestingly, for the Xestospongin $C$ dataset, the data did not achieve statistical significance for mEPSP size $(p=0.10$, one-way ANOVA). This could indicate only weak to no homeostatic pressure in the presence of Xestospongin C. Nevertheless,
EPSPs were significantly reduced (Figures 6C, middle, 6D) because of a marked decrease in quantal content (Figure 6C, right).

Taking all of these data together, for each case where we examined a dual impairment of $\mathrm{RyR}$ and $\mathrm{IP}_{3} \mathrm{R}$ the EPSP amplitudes were all quite low with concomitant VGlut overexpression (Figures 3-6).

\section{PHD in Very Low Extracellular Calcium}

We wondered how impairment of channels that mediate release of calcium from intracellular stores might cause the electrophysiological phenotypes that we observed. It could be the case that they are part of the PHD system. Or it could be the case that impairing these channels does not impinge upon PHD signaling itself-but their loss may sensitize the synapse to additional challenges, such as those brought on by PHD. 
Our prior work suggested that these ER calcium store channels and the signaling systems that control them are required to maintain homeostatic potentiation throughout life (Brusich et al., 2015; James et al., 2019). We also found a related result: impairing $\mathrm{Ca}^{2+}$ store release mollified hyperexcitability phenotypes caused by gain-of-function $\mathrm{Ca}_{\mathrm{V}} 2$ amino-acid substitutions in the alphal subunit Cacophony. $\mathrm{Ca}_{\mathrm{V}} 2$ channels mediate synaptic calcium influx at the NMJ (Brusich et al., 2018). In light of these prior data, we considered two possibilities for PHD. One model is that the $\mathrm{IP}_{3} \mathrm{R}$ and RyR channels play a role in ensuring proper level of neurotransmission coincident with PHD. A different model is that calcium itself plays the important role. If this latter idea were true, it might be the case that lowering calcium influx into the presynaptic terminal would also be sufficient to interact with the PHD signaling process, ultimately lowering evoked transmission.

As a test, we measured release over a range of low extracellular calcium concentrations $(0.2-0.5 \mathrm{mM})$. We examined six genotypes: (1) WT; (2) w; OK371/+; (3) w; VGlut, OK371/+; (4) $w ; R y R^{E 4340 K /+; ~ i t p r} r^{90 B} /+$; (5) $w$; OK371/RyR $R^{E 4340 K}$; itpr $r^{90 B /+;}$ and (6) $w$; VGlut, OK371/RyR ${ }^{E 4340 K}$; itpr ${ }^{90 B} /+$. To organize data and to calculate calcium co-operativity, we plotted quantal content as a function of calcium concentration, with the $x-y$ axes on a log-log scale (Figures 7A,B). To account for different $\mathrm{Ca}^{2+}$ driving forces in the different concentrations, we corrected QC for nonlinear summation in our plots and in our subsequent analyses (NLS Corrected QC; Martin, 1955).

Non-linear regression analyses revealed that there was no significant difference in calcium co-operativity between any of these genotypes over the range of extracellular $\left[\mathrm{Ca}^{2+}\right]$ we tested (Figures 7A,B). The calculated log-log slope values of the control PHD genotypes were: WT $(\log -\log$ slope $=1.810)$, $w ; O K 371 /+(\log -\log$ slope $=1.884)$, and $w ;$ VGlut, OK371/+ (log-log slope $=2.117)$. Comparing those three slopes with one another by nonlinear regression yielded no significant difference in slope $(p=0.91)$. The log-log slope values of the double heterozygous conditions were: $w$; $R y R^{E 4340 K /+;} i \mathrm{tpr}^{90 B} /+$ $(\log -\log$ slope $=1.737), w ; O K 371 / R y R^{E 4340 K} ; i t p r^{90 B} /+(\log -\log$ slope = 2.102), and $w$; VGlut, OK371/RyR ${ }^{E 4340 K} ; i t p r^{90 B} /+(\log -$ $\log$ slope $=1.601)$. Comparing those slopes with one another also yielded no significant difference ( $p=0.77$ ).

Even though there was no significant difference in calcium co-operativity of release over the range of low $\left[\mathrm{Ca}^{2+}\right]$ conditions examined, our data did show a very large drop in release between 0.3 and $0.2 \mathrm{mM}\left[\mathrm{Ca}^{2+}\right]$-specifically for the genotypes where PHD was induced by UAS-VGlut overexpression, or for the genotypes with a double heterozygous impairment of $R y R$ and itpr. Examining the raw data at $0.2 \mathrm{mM}\left[\mathrm{Ca}^{2+}\right]$, we observed that there was significant homeostatic pressure for PHD signified by mEPSP amplitude increases in the
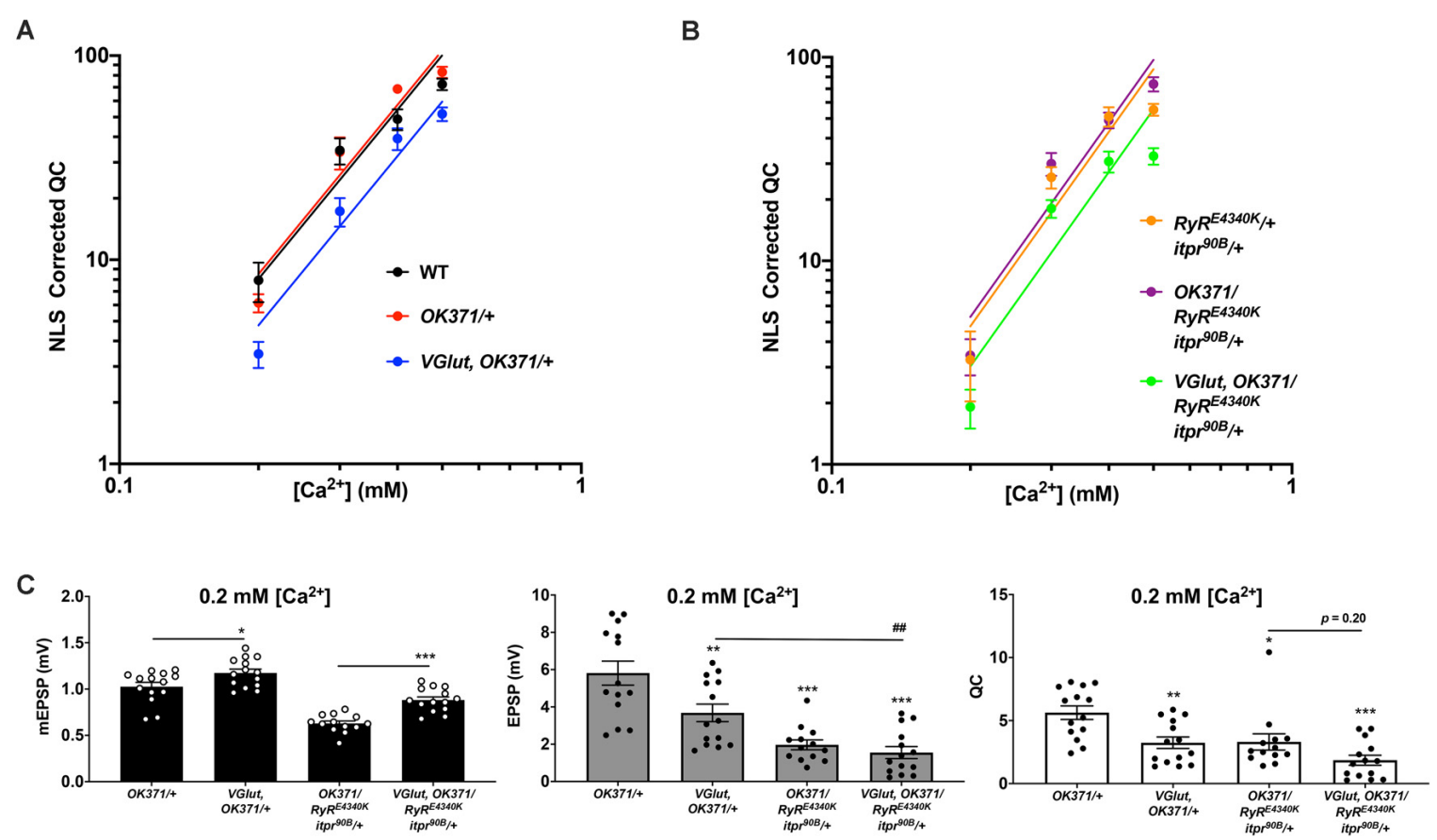

FIGURE 7 | $\mathrm{Ca}^{2+}$ concentration-sensitivity of PHD execution. (A) log-log plots of recording saline $\left[\mathrm{Ca}^{2+}\right]$ vs. QC corrected for non-linear summation for WT, OK371/t, and VG/ut, OK371/+ conditions. Across the range of $\left[\mathrm{Ca}^{2+}\right]$ examined, there is no significant difference in calcium cooperativity for these conditions (Nonlinear Regression, $p=0.91$ ). (B) Data plotted as in (A) but this time with a double heterozygous $R y R^{E 4340 K /+;}$ itpr $908 /+$ genetic background. Across the range of $\left[\mathrm{Ca}^{2+}\right]$ examined, there is no significant difference in calcium cooperativity for these conditions (Nonlinear Regression, $p=0.78$ ). (C) Raw data for mEPSPs (left); raw data for EPSPs (middle); raw data for QC (right). All data are for the indicated NMJ genotypes in $0.2 \mathrm{mM}\left[\mathrm{Ca}^{2+}\right]$; bars are averages and error bars are \pm SEM. For mEPSPs, ${ }^{*} p<0.05$ and ${ }^{* * *} p<0.001$ by Student's $t$-test, comparing PHD-challenged genotypes vs. unchallenged genetic controls. For EPSPs and QC, ${ }^{*} p<0.05$, ${ }^{* *} p<0.01$, and ${ }^{* \star *} p<0.001$ vs. OK371/+; ${ }^{\# \#} p<0.01$; EPSP and QC analyses done across multiple genotypes by one-way ANOVA with Tukey's post-hoc. 
VGlut-overexpression background (Figure 7C, left). Yet except for the control NMJs, EPSP amplitudes were very much diminished (Figure 7C, middle) because of stark drops in QC (Figure 7C, right).

Together, the data point to two conclusions. First, low extracellular calcium on its own appears to be a case where the synapse experiences a synergistic interaction with PHD challenge (Figure 7C, VGlut, OK371/+ data). Second, double heterozygous impairment of $R y R$ and itpr appears to cause very low levels of baseline release in low calcium, irrespective of PHD challenge (Figure 7C, middle; compare with Figure 3C). Taken together, these data suggest that lowering presynaptic calcium by any means (impairing store release and/or impairing influx) is sufficient to impair evoked levels of excitation, in conjunction with a PHD challenge.

\section{PHD Challenge Interacts With Impaired Cav2 Function}

As a final test, we turned back to genetics. Drosophila $\mathrm{Ca}_{\mathrm{V}} 2$ channels mediate synaptic calcium influx at the NMJ. We used a hypomorphic mutant in the $\mathrm{Ca}_{\mathrm{V}} 2$ alpha1 subunitencoding cacophony gene, $\mathrm{cac}^{\mathrm{S}}$, to limit calcium influx. $\mathrm{Ca}_{\mathrm{V}} 2$ is essential for viability, but $\mathrm{cac}^{S}$ hypomorphs are viable and fertile (Smith et al., 1998; Kawasaki et al., 2000). Earlier work showed that the $\mathrm{cac}^{S}$ homozygous condition dampens NMJ
EPSP amplitude by about 70-80\% (Frank et al., 2006); calcium imaging data suggest this is due to a $\sim 50 \%$ decrease in $\mathrm{Ca}^{2+}$ influx during evoked stimulation (Müller and Davis, 2012). Beyond this phenotype in baseline neurotransmission, $\mathrm{cac}^{S}$ hypomorphs also block PHP expression and PHP-associated increases in presynaptic calcium influx (Frank et al., 2006; Müller and Davis, 2012).

With a single cross, we generated hemizygous $\mathrm{cac}^{\mathrm{S}} / Y$; VGlut, OK371/+ male larvae (Figure 8A). Compared to $\mathrm{cac}^{S} / Y$ as a baseline mutant control, $\mathrm{cac}^{\mathrm{S}} / Y$; VGlut, OK371/+ NMJs have a marked increase in mEPSP size (Figure 8B), indicating homeostatic pressure to induce PHD (Figure 8B). However, comparing evoked potentials of those two conditions shows that $\mathrm{cac}^{S} / Y$; VGlut, OK371/+ NMJs have much smaller EPSPs (Figure 8C) and a very large decrease in QC (Figure 8D).

\section{DISCUSSION}

We began this study in search of genetic conditions that affect PHD (Figure 2). While we did not find any conditions that result in a block of PHD, we did find conditions that provide insight into how calcium regulation may interact with this form of homeostatic plasticity to affect synapse function. When $\mathrm{IP}_{3} \mathrm{R}$ and RyR functions are partially impaired-either by genetics or by pharmacology-the NMJ still executes a PHD-like
A
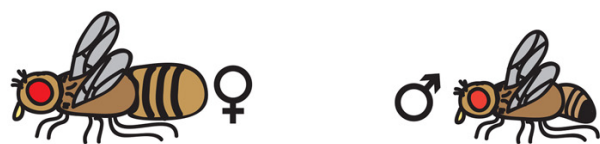

$\mathrm{cac}^{\mathrm{s}} / \mathrm{cac}^{\mathrm{s}}$ X w/Y; UAS-VGlut, OK371-Gal4/(Bal)

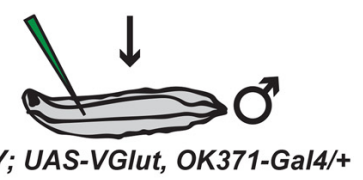

cac $^{S} / Y$; UAS-VGlut, OK371-Gal4/+

C

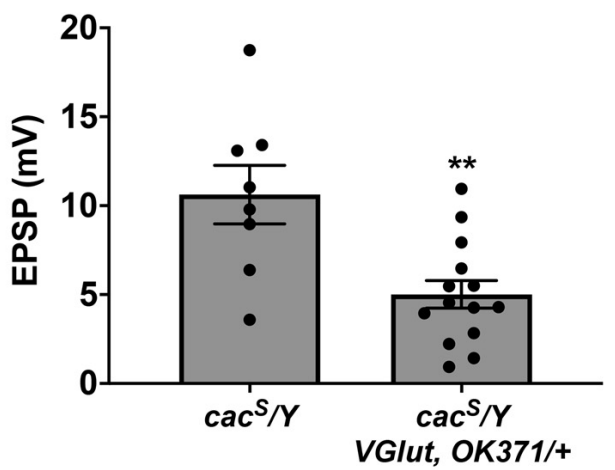

B

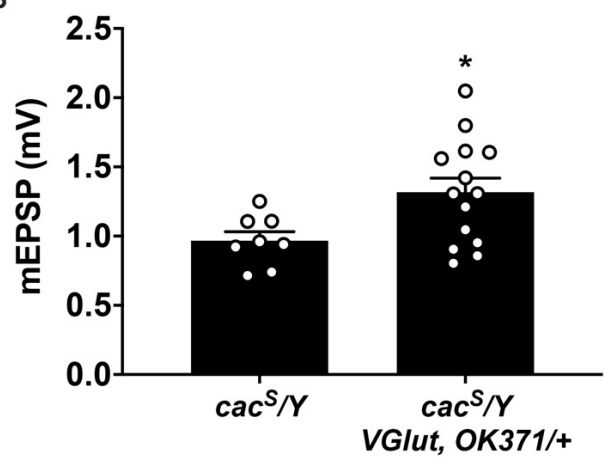

D

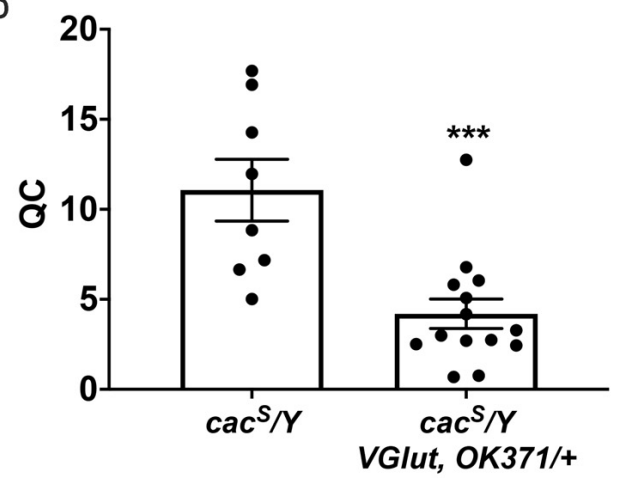

FIGURE 8 | Partial impairment of Cav2/Cacophony and PHD. (A) Crossing scheme for generating larvae for electrophysiological recording. Male larvae were hemizygous for the $\mathrm{cac}^{\mathrm{S}}$ hypomorphic mutation. Fly artwork reproduced from Brusich et al. (2015) under a Creative Commons Attribution License. (B) Raw data for mEPSPs. (C) Raw data for EPSPS. (D) Raw data for QC. For (B-D), bars are averages and error bars are \pm SEM. ${ }^{\star} p<0.05$, ${ }^{\star \star} p<0.01$, and ${ }^{\star \star \star} p<0.001$ by Student's $t$-test comparing the control $\mathrm{cac}^{S}$ dataset (no VGlut overexpression) vs. the experimental cac ${ }^{S}$ dataset (VGlut overexpression). 
process. But that process goes beyond what is appropriate for the homeostatic pressure that is applied to the system. As a result, evoked potentials at the NMJ are much smaller than baseline (Figures 3-6). A similar phenotype is observed when extracellular $\left[\mathrm{Ca}^{2+}\right]$ is lowered to $0.2 \mathrm{mM}$ (Figure 7) and when the $\mathrm{Ca}_{\mathrm{V}} 2$ alpha1 subunit gene cacophony harbors a hypomorphic mutation, $\mathrm{cac}^{S}$ (Figure 8).

This phenotype has important implications for proper control of synapse function. Taking our data together, we propose that perturbations that dampen calcium efflux from stores or perturbations that dampen calcium influx from the extracellular environment can both synergistically interact with a PHD challenge to control levels of evoked neurotransmission (Figure 9).

\section{Screen Limitations}

We did not identify conditions that blocked PHD, and here we discuss potential limitations of the screen. First, our primary assay was electrophysiology, and we employed a candidate-based method, similar to what has previously been documented in the field for PHP (Frank et al., 2020). By definition, candidatebased screens are limited in scope. Second, we focused on factors previously implicated in the maintenance of PHP function (or closely related signaling factors). The idea was that some factors needed to maintain synaptic homeostasis may be needed to orient the NMJ toward a proper, physiological level of function, regardless of the nature of the homeostatic challenge. This idea could have valence, but it was not guaranteed to produce mutant conditions with greater than normal evoked amplitudes in our screen.

Regarding the electrophysiological data, we did find instances in which the screened EPSP was numerically larger than the baseline for VGlut, OK371/+, but no instances identified as "PHD-blocking" (Figure 2). The VGlut, OK371/+ baseline evoked potential was high $(\sim 35 \mathrm{mV})$, so it is possible that potential positives at a higher potential could be obscured by the limits of non-linear summation. There were also variations from line to line in resting membrane potential, input resistance, and the degree of $\mathrm{mEPSP}$ increase indicative of $\mathrm{PHD}$ challenge (Supplementary Table 1). All of these parameters could contribute to false negatives for the screen. Unless a screen is done to saturation, there will be false negatives. It is important to interpret those parsimoniously. For our screen, we believe the way to interpret a negative is not to state that the screen definitively ruled out a factor-rather, the screen failed to rule in that factor for follow-up study.

\section{Similarities and Differences With Prior PHD Studies at the NMJ}

We were able to conduct a PHD screen using our recombinant stock with the UAS-VGlut and OK371-Gal4 elements on the same chromosome. In principle, such a stock can pick up modifier mutations. The trade-off was a simplified, single-generation crossing scheme for genetic screens. Our recombinant stock with the driver and UAS elements in cis maintains consistent PHD challenge from generation to generation, and it behaves similarly electrophysiologically to trans OK371/VGlut combinations used in other studies (Daniels et al., 2004; Gaviño et al., 2015; Li et al., 2018).

There are differences between our study and the findings of other published work. Prior studies have used WT (or $w^{1118}$ ) as a control background when compared to VGlut overexpression (Daniels et al., 2004; Gaviño et al., 2015; Li et al., 2018). This is a standard practice. Those studies reported precise PHD when comparing WT vs. OK371/VGlut third instar larvae-decreased QC at OK371/VGlut NMJs resulting in unchanged evoked transmission. We replicated this finding (Figure 1). However, we also used our Gal4 driver stock background OK371/+ as an additional control. For that comparison, we saw a slight depression in the evoked amplitude of OK371, VGlut/+ NMJs (Figure 1). One possibility is that our recombinant stock was acting as a sensitized background.

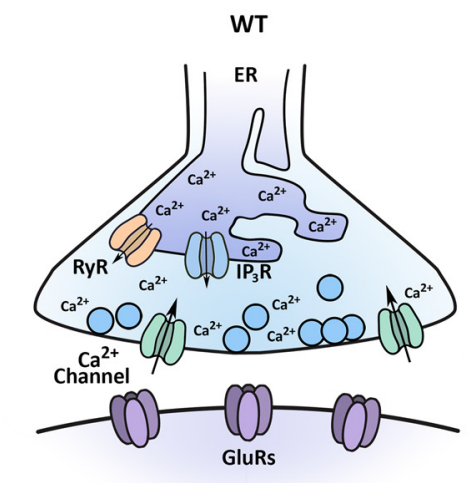

Evoked potentials normal

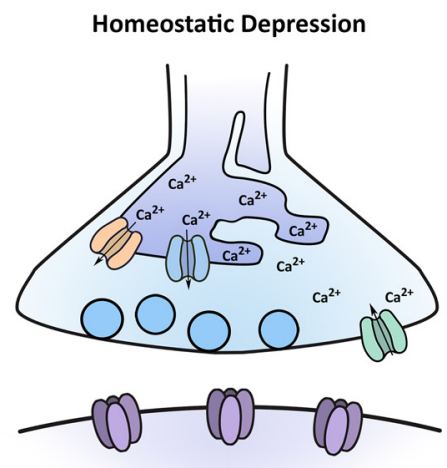

Evoked potentials $\approx$ normal

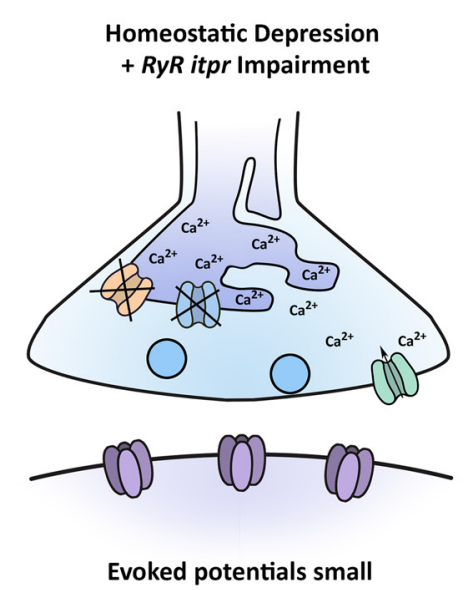

Evoked potentials small

FIGURE 9 | Model for how multiple calcium sources interact with the process of PHD. Under baseline conditions, Cav2-type calcium channels contribute to synapse function, as may RyRs and $\mathrm{IP}_{3} \mathrm{Rs}$. Under conditions inducing PHD, synaptic vesicles are enlarged, and $\mathrm{QC}$ is decreased, through regulation of sources of calcium. When PHD challenge is coupled with concomitant impairment of RyR and $\mathrm{P}_{3} \mathrm{R}$ channels, evoked potentials are significantly diminished. 
A second difference comes from the low extracellular calcium test. A low extracellular calcium experiment was previously done when VGlut overexpression was first characterized (Daniels et al., 2004). For that study, the authors showed that QC was significantly diminished compared to wild-type NMJs by the method of failure analysis. Taking the data of that study in aggregate, the authors concluded that PHD was intact in a variety of conditions, including saline with very low extracellular $\left[\mathrm{Ca}^{2+}\right]\left(0.23 \mathrm{mM} \mathrm{Ca}{ }^{2+}, 20 \mathrm{mM} \mathrm{Mg}^{2+}\right)$. Our study may appear to conflict with that study because we found that saline with very low $\left[\mathrm{Ca}^{2+}\right]\left(0.2 \mathrm{mM} \mathrm{Ca}^{2+}, 10 \mathrm{mM} \mathrm{Mg}^{2+}\right)$ is conducive to an interaction with PHD, resulting in low evoked release. One possibility is that since the original study was examining failure percentage vs. WT-and not the absolute value of mEPSPs or EPSPs in low calcium, this might not be as easily observed. Other differences might be attributed to genetic background or other differences in recording saline, like magnesium concentration.

Finally, one other study previously examined the effects of a $\mathrm{cac}^{S}$ mutation with concomitant VGlut overexpression (Gaviño et al., 2015). The authors did not find the low evoked potentials that we report. The major difference between that experiment and ours is that the prior work examined the $\mathrm{cac}^{S}$ mutation in an extracellular $\left[\mathrm{Ca}^{2+}\right](1.0 \mathrm{mM})$ that was double that of our study. The result was a $\mathrm{Ca}^{2+}$ driving force that yielded robust baseline EPSPs, even in the $\mathrm{cac}^{S}$ mutant background (Gaviño et al., 2015). Given our results with low calcium concentration (Figure 7), a similar effect may be at work here.

\section{Known Roles for Calcium in Controlling Homeostatic Plasticity}

The notion that calcium contributes to successful homeostatic signaling is not new. Many roles for voltage-gated calcium channels in synaptic homeostasis are well-documented (Frank, 2014a,b). Prior to our study, there was evidence for voltagegated calcium channel regulation for both NMJ PHP and PHD. For PHP, loss-of-function conditions in $\mathrm{Ca}_{\mathrm{V}} 2 /$ cacophony can impair or block this form of homeostatic regulation (Frank et al., 2006, 2009; Müller and Davis, 2012; Spring et al., 2016). Calcium imaging experiments suggest that the reason is because an increase in calcium influx through $\mathrm{Ca}_{V} 2$ is required for the upregulation of quantal content during PHP, and mutant conditions like $c a c^{S}$ block this increase (Müller and Davis, 2012). Recent studies report that Cacophony and other active zone protein levels increase at the NMJ active zone in response to PHP homeostatic challenges (Böhme et al., 2019; Goel et al., 2019; Gratz et al., 2019). And work from mammalian systems mirrors these findings. For example, with mouse hippocampal cultures, TTX exposure induces a homeostatic decrease in presynaptic calcium influx (Zhao et al., 2011).

The converse appears true for PHD. Calcium imaging data from two different studies has shown a decrease in the size of calcium transients at the NMJ in response to presynaptic nerve firing in VGlut-overexpressing animals (Gaviño et al., 2015; Li et al., 2018). The data are mixed on how these decreased transients might come about during PHD. Using a tagged UAS-cacophony cDNA transgene, two studies verified that there was a reduction in the amount of GFP-tagged Cacophony alpha1 subunits in $\mathrm{Ca}_{V} 2$ in a VGlut-overexpressing background (Gaviño et al., 2015; Gratz et al., 2019). However, one of these same studies demonstrated that if a tagged genomic construct is used instead, that same $\mathrm{Ca}_{V} 2$ reduction is not observed (Gratz et al., 2019). Since the transgenic tagged Cacophony-GFP is the product of a single cac splice isoform (Kawasaki et al., $2002,2004)$, it could be the case that some isoforms are more dynamically trafficked at the synapse. Another possibility is that existing active zone components are somehow modulated during PHD. Regardless of the actual mechanism, the phenomenon appears conserved: again, with rodent hippocampal preparations, increased neuronal activity through gabazine exposure induces a PHD-like phenomenon ultimately resulting in decreases in calcium influx and release (Zhao et al., 2011; Jeans et al., 2017).

\section{How Do Calcium Stores Interact With PHD?}

Calcium stores have been studied in the context of neurotransmission and plasticity. We know that endoplasmic reticulum (ER) can be visualized at Drosophila NMJ terminals (Summerville et al., 2016), and recently developed imaging tools employed in multiple systems (including at the Drosophila NMJ) show how nerve stimulation results in dynamic changes to ER lumenal calcium (de Juan-Sanz et al., 2017; Handler et al., 2019; Oliva et al., 2020). In parallel, other groups working at the Drosophila NMJ have demonstrated important roles in baseline neurotransmission and in PHP for ER resident proteins (Genç et al., 2017; Kikuma et al., 2017). And from our prior work, we know that store calcium channels and upstream signaling components are important for maintaining the NMJ's capacity for PHP throughout life (Brusich et al., 2015; James et al., 2019). We also know that disrupting these same factors can ameliorate hyperexcitability associated with gains of $\mathrm{Ca}_{V} 2$ function (Brusich et al., 2018). Finally, from mammalian work it is clear that $\mathrm{IP}_{3} \mathrm{Rs}$, RyRs, and intracellular calcium govern a variety of forms of neuroplasticity (Berridge, 2016), including paired pulse facilitation (Emptage et al., 2001), and modulation of voltage-gated calcium channel activity (Lee et al., 2000; Catterall, 2011).

If PHD were simply a matter of properly functioning neurotransmission machinery, then it is not entirely obvious why PHD would be so sensitive to the amount of calcium available such that evoked release would be impaired greatly either when store-operated channels were impaired or when the amount of influx was lowered. In our study, neurotransmission has not been lowered beyond a point of synapse failure. This means that there is still functional machinery. And $\mathrm{PHD}$, per se, is not disrupted-indeed, there is still depression.

With any type of homeostatic system, there not only needs to be error detection (large quantal size) and correction (decreased quantal content), but there also need to be brakes applied to the system to prevent some kind of overcorrection. At first glance, our data could suggest some manner of PHD "overcorrection." In our view, this is an interesting and understudied type of phenomenon that could be examined in many homeostatic systems. But it is also true that the nature of the PHD challenge 
could simply represent a genetic background that renders the synapse sensitive to any additional insults.

So how exactly do levels of calcium (or the function of distinct types of calcium channels found at the synapse) ultimately affect excitation levels? This is a difficult problem. The first step might be to narrow the relevant tissue type(s) involved in PHD signaling. ER and store-operated channels are relevant to the functions of many tissues. In principle, our genetic loss-of-function manipulations to itpr and $R y R$ could affect store-operated channels either in the neuron or in the muscle or in surrounding tissues like glia. Our pharmacological manipulations using Dantrolene and Xestospongin C could also affect multiple tissue types. Therefore, in principle, changing the levels of cytosolic calcium could either affect local signaling in the neuron, or it could result in aberrant signaling back to the presynaptic neuron, disorienting the homeostat.

We favor the idea that the relevant calcium signal is local in the motor neuron for two reasons. First, from our own data, we were able to observe the small evoked neurotransmission phenotype either with manipulations to store calcium or with manipulations that affect presynaptic calcium influx, including partial loss-of-function of neuronal cacophony. Second, a recent study puts forth data suggesting that when VGlut overexpression induces PHD, this happens exclusively because of excess presynaptic glutamate release, and presynaptic depression is initiated independent of any sort of postsynaptic response (Li et al., 2018). Such an autocrine signaling mechanism could very well reveal a role for intracellular calcium signaling in the presynapse.

\section{DATA AVAILABILITY STATEMENT}

The raw data supporting the conclusions of this article will be made available by the authors, without undue reservation.

\section{REFERENCES}

Berridge, M. J. (1984). Inositol trisphosphate and diacylglycerol as second messengers. Biochem. J. 220, 345-360. doi: 10.1042/bj22 00345

Berridge, M. J. (1987). Inositol trisphosphate and diacylglycerol: two interacting second messengers. Annu. Rev. Biochem. 56, 159-193. doi: 10.1146/annurev.bi. 56.070187 .001111

Berridge, M. J. (1998). Neuronal calcium signaling. Neuron 21, 13-26. doi: 10.1016/s0896-6273(00)80510-3

Berridge, M. J. (2016). The inositol trisphosphate/calcium signaling pathway in health and disease. Physiol. Rev. 96, 1261-1296. doi: 10.1152/physrev.000 06.2016

Böhme, M. A., McCarthy, A. W., Grasskamp, A. T., Beuschel, C. B., Goel, P., Jusyte, M., et al. (2019). Rapid active zone remodeling consolidates presynaptic potentiation. Nat. Commun. 10:1085. doi: 10.1038/s41467-019-08977-6

Brusich, D. J., Spring, A. M., and Frank, C. A. (2015). A single-cross, RNA interference-based genetic tool for examining the long-term maintenance of homeostatic plasticity. Front. Cell. Neurosci. 9:107. doi: 10.3389/fncel.2015. 00107

Brusich, D. J., Spring, A. M., James, T. D., Yeates, C. J., Helms, T. H., Frank, C. A., et al. (2018). Drosophila $\mathrm{CaV} 2$ channels harboring human migraine mutations cause synapse hyperexcitability that can be suppressed by inhibition of a $\mathrm{Ca}^{2+}$

\section{AUTHOR CONTRIBUTIONS}

CJY and CAF designed research, performed research, analyzed the data, wrote, and edited the article. All authors contributed to the article and approved the submitted version.

\section{FUNDING}

Funding supporting this work includes an NSF Grant (1557792) and an NIH/NINDS Grant (R01NS085164) to CAF. CJY was supported by an NIH/NINDS Predoctoral Training Grant to the University of Iowa (UI) Interdisciplinary Graduate Program in Neuroscience (T32NS007421-PI Daniel $\mathrm{T}$. Tranel), as well as a post-comprehensive exam predoctoral summer fellowship and a Ballard and Seashore Dissertation fellowship via the Graduate College at UI.

\section{ACKNOWLEDGMENTS}

We thank members of the Frank lab for helpful comments. We thank the laboratories of Drs. Tina Tootle, Fang Lin, Toshihiro Kitamoto, Pamela Geyer, and Lori Wallrath for helpful discussions. We also thank Drs. Toshihiro Kitamoto, Joshua Weiner, Christopher Stipp, and Mark Stamnes for helpful feedback on an earlier written version of this study. An earlier version of this manuscript was released as a pre-print at bioRxiv (Yeates and Frank, 2020).

\section{SUPPLEMENTARY MATERIAL}

The Supplementary Material for this article can be found online at: https://www.frontiersin.org/articles/10.3389/fncel. 2021.618393/full\#supplementary-material.

store release pathway. PLoS Genetics 14:e1007577. doi: 10.1371/journal.pgen 1007577

Budnik, V., Koh, Y. H., Guan, B., Hartmann, B., Hough, C., Woods, D., et al. (1996). Regulation of synapse structure and function by the Drosophila tumor suppressor gene dlg. Neuron 17, 627-640. doi: 10.1016/s0896-6273(00)80196-8

Catterall, W. A. (2011). Voltage-gated calcium channels. Cold Spring Harb. Perspect. Biol. 3:a003947. doi: 10.1101/cshperspect.a003947

Cull-Candy, S. G., Miledi, R., Trautmann, A., and Uchitel, O. D. (1980). On the release of transmitter at normal, myasthenia gravis and myasthenic syndrome affected human end-plates. J. Physiol. 299, 621-638. doi: 10.1113/jphysiol.1980. sp013145

Daniels, R. W., Collins, C. A., Gelfand, M. V., Dant, J., Brooks, E. S., Krantz, D. E., et al. (2004). Increased expression of the Drosophila vesicular glutamate transporter leads to excess glutamate release and a compensatory decrease in quantal content. J. Neurosci. 24, 10466-10474. doi: 10.1523/JNEUROSCI.300104.2004

Davis, G. W. (2013). Homeostatic signaling and the stabilization of neural function. Neuron 80, 718-728. doi: 10.1016/j.neuron.2013.09.044

Davis, G. W., and Müller, M. (2015). Homeostatic control of presynaptic neurotransmitter release. Annu. Rev. Physiol. 77, 251-270. doi: 10.1146/annurev-physiol-021014-071740

Davis, G. W., DiAntonio, A., Petersen, S. A., and Goodman, C. S. (1998). Postsynaptic PKA controls quantal size and reveals a retrograde signal that 
regulates presynaptic transmitter release in Drosophila. Neuron 20, 305-315. doi: 10.1016/s0896-6273(00)80458-4

de Juan-Sanz, J., Holt, G. T., Schreiter, E. R., de Juan, F., Kim, D. S., Ryan, T. A., et al. (2017). Axonal endoplasmic reticulum $\mathrm{Ca}^{2+}$ content controls release probability in CNS nerve terminals. Neuron 93, 867-881.e866.doi: 10.1016/j. neuron.2017.01.010

Delvendahl, I., and Müller, M. (2019). Homeostatic plasticity-a presynaptic perspective. Curr. Opin. Neurobiol. 54, 155-162. doi: 10.1016/j.conb.2018. 10.003

Dockendorff, T. C., Robertson, S. E., Faulkner, D. L., and Jongens, T. A. (2000). Genetic characterization of the 44D-45B region of the Drosophila melanogaster genome based on an F2 lethal screen. Mol. Gen. Genet. 263, 137-143. doi: $10.1007 / \mathrm{s} 004380050040$

Emptage, N. J., Reid, C. A., and Fine, A. (2001). Calcium stores in hippocampal synaptic boutons mediate short-term plasticity, store-operated $\mathrm{Ca}^{2+}$ entry and spontaneous transmitter release. Neuron 29, 197-208. doi: 10.1016/s08966273(01)00190-8

Frank, C. A. (2014a). Homeostatic plasticity at the Drosophila neuromuscular junction. Neuropharmacology 78, 63-74. doi: 10.1016/j.neuropharm.2013. 06.015

Frank, C. A. (2014b). How voltage-gated calcium channels gate forms of homeostatic synaptic plasticity. Front. Cell. Neurosci. 8:40. doi: 10.3389/fncel. 2014.00040

Frank, C. A., James, T. D., and Muller, M. (2020). Homeostatic control of Drosophila neuromuscular junction function. Synapse 74:e22133. doi: 10.1002/syn.22133

Frank, C. A., Kennedy, M. J., Goold, C. P., Marek, K. W., and Davis, G. W. (2006). Mechanisms underlying the rapid induction and sustained expression of synaptic homeostasis. Neuron 52, 663-677. doi: 10.1016/j.neuron.2006. 09.029

Frank, C. A., Pielage, J., and Davis, G. W. (2009). A presynaptic homeostatic signaling system composed of the Eph receptor, ephexin, Cdc42 and CaV2.1 calcium channels. Neuron 61, 556-569. doi: 10.1016/j.neuron.2008. 12.028

Gafni, J., Munsch, J. A., Lam, T. H., Catlin, M. C., Costa, L. G., Molinski, T. F., et al. (1997). Xestospongins: potent membrane permeable blockers of the inositol 1,4,5-trisphosphate receptor. Neuron 19, 723-733. doi: 10.1016/s08966273(00)80384-0

Gaviño, M. A., Ford, K. J., Archila, S., and Davis, G. W. (2015). Homeostatic synaptic depression is achieved through a regulated decrease in presynaptic calcium channel abundance. eLife 4:e05473. doi: 10.7554/eLife.05473

Genç, Ö., Dickman, D. K., Ma, W., Tong, A., Fetter, R. D., Davis, G. W., et al. (2017). MCTP is an ER-resident calcium sensor that stabilizes synaptic transmission and homeostatic plasticity. eLife 6:e22904. doi: 10.7554/eLife. 22904

Goel, P., Dufour Bergeron, D., Bohme, M. A., Nunnelly, L., Lehmann, M., Buser, C., et al. (2019). Homeostatic scaling of active zone scaffolds maintains global synaptic strength. J. Cell. Biol. 218, 1706-1724. doi: 10.1083/jcb. 201807165

Gratz, S. J., Goel, P., Bruckner, J. J., Hernandez, R. X., Khateeb, K., Macleod, G. T., et al. (2019). Endogenous tagging reveals differential regulation of $\mathrm{ca}^{2+}$ channels at single active zones during presynaptic homeostatic potentiation and depression. J. Neurosci. 39, 2416-2429. doi: 10.1523/JNEUROSCI.306818.2019

Handler, A., Graham, T. G. W., Cohn, R., Morantte, I., Siliciano, A. F., Zeng, J., et al. (2019). Distinct dopamine receptor pathways underlie the temporal sensitivity of associative learning. Cell 178, 60-75.e19.doi: 10.1016/j.cell.2019. 05.040

Hazelrigg, T., Levis, R., and Rubin, G. M. (1984). Transformation of white locus DNA in drosophila: dosage compensation, zeste interaction and position effects. Cell 36, 469-481. doi: 10.1016/0092-8674(84)90240-x

James, T. D., Zwiefelhofer, D. J., and Frank, C. A. (2019). Maintenance of homeostatic plasticity at the Drosophila neuromuscular synapse requires continuous IP3-directed signaling. eLife 8:e39643. doi: 10.7554/eLife.39643

Jeans, A. F., van Heusden, F. C., Al-Mubarak, B., Padamsey, Z., and Emptage, N. J. (2017). Homeostatic presynaptic plasticity is specifically regulated by P/Q-type $\mathrm{Ca}^{2+}$ channels at mammalian hippocampal synapses. Cell Rep. 21, 341-350. doi: 10.1016/j.celrep.2017.09.061
Kawasaki, F., Collins, S. C., and Ordway, R. W. (2002). Synaptic calcium-channel function in Drosophila: analysis and transformation rescue of temperaturesensitive paralytic and lethal mutations of cacophony. J. Neurosci. 22, 5856-5864. doi: 10.1523/JNEUROSCI.22-14-05856.2002

Kawasaki, F., Felling, R., and Ordway, R. W. (2000). A temperature-sensitive paralytic mutant defines a primary synaptic calcium channel in Drosophila. J. Neurosci. 20, 4885-4889. doi: 10.1523/JNEUROSCI.20-13-04885.2000

Kawasaki, F., Zou, B., Xu, X., and Ordway, R. W. (2004). Active zone localization of presynaptic calcium channels encoded by the cacophony locus of Drosophila. J. Neurosci. 24, 282-285. doi: 10.1523/JNEUROSCI.3553-03.2004

Kikuma, K., Li, X., Kim, D., Sutter, D., and Dickman, D. K. (2017). Extended synaptotagmin localizes to presynaptic ER and promotes neurotransmission and synaptic growth in Drosophila. Genetics 207, 993-1006. doi: 10.1534/genetics.117.300261

Lee, A., Scheuer, T., and Catterall, W. A. (2000). $\mathrm{Ca}^{2+} /$ calmodulin-dependent facilitation and inactivation of $\mathrm{P} / \mathrm{Q}$-type $\mathrm{Ca}^{2+}$ channels. J. Neurosci. 20, 6830-6838. doi: 10.1523/JNEUROSCI.20-18-06830.2000

Lefebvre, S., Burglen, L., Reboullet, S., Clermont, O., Burlet, P., Viollet, L., et al. (1995). Identification and characterization of a spinal muscular atrophydetermining gene. Cell 80, 155-165. doi: 10.1016/0092-8674(95)90460-3

Li, X., Goel, P., Wondolowski, J., Paluch, J., and Dickman, D. (2018). A glutamate homeostat controls the presynaptic inhibition of neurotransmitter release. Cell Rep. 23, 1716-1727. doi: 10.1016/j.celrep.2018.03.130

Mahr, A., and Aberle, H. (2006). The expression pattern of the Drosophila vesicular glutamate transporter: a marker protein for motoneurons and glutamatergic centers in the brain. Gene Expr. Patterns 6, 299-309. doi: 10.1016/j.modgep. 2005.07.006

Marder, E., and Goaillard, J. M. (2006). Variability, compensation and homeostasis in neuron and network function. Nat. Rev. Neurosci. 7, 563-574. doi: $10.1038 / \mathrm{nrn} 1949$

Marie, B., Pym, E., Bergquist, S., and Davis, G. W. (2010). Synaptic homeostasis is consolidated by the cell fate gene gooseberry, a Drosophila pax $3 / 7$ homolog. J. Neurosci. 30, 8071-8082. doi: 10.1523/JNEUROSCI.5467-09.2010

Martin, A. R. (1955). A further study of the statistical composition on the end-plate potential. J. Physiol. 130, 114-122. doi: 10.1113/jphysiol.1955. sp005397

Meyer, F., and Aberle, H. (2006). At the next stop sign turn right: the metalloprotease Tolloid-related 1 controls defasciculation of motor axons in Drosophila. Development 133, 4035-4044. doi: 10.1242/dev.02580

Müller, M., and Davis, G. W. (2012). Transsynaptic control of presynaptic $\mathrm{Ca}^{2+}$ influx achieves homeostatic potentiation of neurotransmitter release. Curr. Biol. 22, 1102-1108. doi: 10.1016/j.cub.2012.04.018

Newman, Z. L., Hoagland, A., Aghi, K., Worden, K., Levy, S. L., Son, J. H., et al. (2017). Input-specific plasticity and homeostasis at the Drosophila larval neuromuscular junction. Neuron 93, 1388-1404.e1310. doi: 10.1016/j.neuron. 2017.02.028

Oliva, M. K., Perez-Moreno, J. J., O'Shaughnessy, J., Wardill, T. J., and O'Kane, C. J. (2020). Endoplasmic reticulum lumenal indicators in Drosophila reveal effects of HSP-related mutations on endoplasmic reticulum calcium dynamics. Front. Neurosci. 14:816. doi: 10.3389/fnins.2020.00816

Petersen, S. A., Fetter, R. D., Noordermeer, J. N., Goodman, C. S., and DiAntonio, A. (1997). Genetic analysis of glutamate receptors in Drosophila reveals a retrograde signal regulating presynaptic transmitter release. Neuron 19, 1237-1248. doi: 10.1016/s0896-6273(00)80415-8

Pozo, K., and Goda, Y. (2010). Unraveling mechanisms of homeostatic synaptic plasticity. Neuron 66, 337-351. doi: 10.1016/j.neuron.2010.04.028

Raimer, A. C., Singh, S. S., Edula, M. R., Paris-Davila, T., Vandadi, V., Spring, A. M., et al. (2020). Temperature-sensitive spinal muscular atrophycausing point mutations lead to SMN instability, locomotor defects and premature lethality in Drosophila. Dis. Models Mech. 13:dmm043307. doi: $10.1242 / \mathrm{dmm} .043307$

Sen, A., Yokokura, T., Kankel, M. W., Dimlich, D. N., Manent, J., Sanyal, S., et al. (2011). Modeling spinal muscular atrophy in Drosophila links Smn to FGF signaling. J. Cell. Biol. 192, 481-495. doi: 10.1083/jcb.2010 04016

Simkus, C. R., and Stricker, C. (2002). The contribution of intracellular calcium stores to mEPSCs recorded in layer II neurones of rat barrel cortex. J. Physiol. 545, 521-535. doi: 10.1113/jphysiol.2002.022103 
Smith, L. A., Peixoto, A. A., Kramer, E. M., Villella, A., and Hall, J. C. (1998). Courtship and visual defects of cacophony mutants reveal functional complexity of a calcium-channel alphal subunit in Drosophila. Genetics 149, 1407-1426. Available online at: https://www.genetics.org/content/149/3/1407.

Spring, A. M., Brusich, D. J., and Frank, C. A. (2016). C-terminal Src kinase gates homeostatic synaptic plasticity and regulates fasciclin II expression at the Drosophila neuromuscular junction. PLoS Genet. 12:e1005886. doi: 10.1371/journal.pgen.1005886

Spring, A. M., Raimer, A. C., Hamilton, C. D., Schillinger, M. J., and Matera, A. G. (2019). Comprehensive modeling of spinal muscular atrophy in Drosophila melanogaster. Front. Mol. Neurosci. 12:113. doi: 10.3389/fnmol.2019.00113

Summerville, J. B., Faust, J. F., Fan, E., Pendin, D., Daga, A., Formella, J., et al. (2016). The effects of ER morphology on synaptic structure and function in Drosophila melanogaster. J. Cell. Sci. 129, 1635-1648. doi: 10.1242/jcs. 184929

Turrigiano, G. G. (2008). The self-tuning neuron: synaptic scaling of excitatory synapses. Cell 135, 422-435. doi: 10.1016/j.cell.2008.10.008

Vazquez-Martinez, O., Canedo-Merino, R., Diaz-Munoz, M., and RiesgoEscovar, J. R. (2003). Biochemical characterization, distribution and phylogenetic analysis of Drosophila melanogaster ryanodine and IP3 receptors and thapsigargin-sensitive $\mathrm{Ca}^{2+}$ ATPase. J. Cell Sci. 116, 2483-2494. doi: $10.1242 /$ jcs. 00455

Venkatesh, K., and Hasan, G. (1997). Disruption of the IP3 receptor gene of Drosophila affects larval metamorphosis and ecdysone release. Curr. Biol. 7, 500-509. doi: 10.1016/s0960-9822(06)00221-1

Wagh, D. A., Rasse, T. M., Asan, E., Hofbauer, A., Schwenkert, I., Durrbeck, H., et al. (2006). Bruchpilot, a protein with homology to ELKS/CAST, is required for structural integrity and function of synaptic active zones in Drosophila. Neuron 49, 833-844. doi: 10.1016/j.neuron.2006.02.008

Wang, X., Pinter, M. J., and Rich, M. M. (2016). Reversible recruitment of a homeostatic reserve pool of synaptic vesicles underlies rapid homeostatic plasticity of quantal content. J. Neurosci. 36, 828-836. doi: 10.1523/JNEUROSCI.3786-15.2016

Wilcox, R. A., Primrose, W. U., Nahorski, S. R., and Challiss, R. A. (1998). New developments in the molecular pharmacology of the myo-inositol 1,4,5-trisphosphate receptor. Trends Pharmacol. Sci. 19, 467-475. doi: 10.1016/s0165-6147(98)01260-7

Yeates, C. J., and Frank, C. A. (2020). A class of synaptic signaling molecules required for homeostatic potentiation also tunes homeostatic depression. bioRxiv [Preprint]. doi: 10.1101/2020.10.12.336883

Yeates, C. J., Zwiefelhofer, D. J., and Frank, C. A. (2017). The maintenance of synaptic homeostasis at the Drosophila neuromuscular junction is reversible and sensitive to high temperature. eNeuro 4:ENEURO.0220-17.2017. doi: 10.1523/ENEURO.0220-17.2017

Zhao, C., Dreosti, E., and Lagnado, L. (2011). Homeostatic synaptic plasticity through changes in presynaptic calcium influx. J. Neurosci. 31, 7492-7496. doi: 10.1523/JNEUROSCI.6636-10.2011

Zhao, F., Li, P., Chen, S. R., Louis, C. F., and Fruen, B. R. (2001). Dantrolene inhibition of ryanodine receptor $\mathrm{Ca}^{2+}$ release channels. Molecular mechanism and isoform selectivity. J. Biol. Chem. 276, 13810-13816. doi: 10.1074/jbc. M006104200

Conflict of Interest: The authors declare that the research was conducted in the absence of any commercial or financial relationships that could be construed as a potential conflict of interest.

Copyright (c) 2021 Yeates and Frank. This is an open-access article distributed under the terms of the Creative Commons Attribution License (CC BY). The use, distribution or reproduction in other forums is permitted, provided the original author(s) and the copyright owner(s) are credited and that the original publication in this journal is cited, in accordance with accepted academic practice. No use, distribution or reproduction is permitted which does not comply with these terms. 